Reanalyse und

\title{
Grammatikalisierung in den romanischen Sprachen
}

Herausgegeben von

Jürgen Lang und Ingrid Neumann-Holzschuh

Max Niemeyer Verlag

Tübingen 1999 
Die Deutsche Bibliothek - CIP-Einheitsaufnahme

Reanalyse und Grammatikalisierung in den romanischen Sprachen / hrsg. von Jürgen Lang und Ingrid Neumann-Holzschuh. - Tübingen : Niemeyer, 1999

(Linguistische Arbeiten ; 410)

ISBN 3-484-30410-3 ISSN 0344-6727

(c) Max Niemeyer Verlag GmbH, Tübingen 1999

Das Werk einschließlich aller seiner Teile ist urheberrechtlich geschützt. Jede Verwertung außerhalb der engen Grenzen des Urheberrechtsgesetzes ist ohne Zustimmung des Verlages unzulässig und strafbar. Das gilt insbesondere für Vervielfältigungen, Übersetzungen, Mikroverfilmungen und die Einspeicherung und Verarbeitung in elektronischen Systemen. Printed in Germany.

Gedruckt auf alterungsbeständigem Papier.

Druck: Weihert-Druck GmbH, Darmstadt

Buchbinder: Nädele Verlags- und Industriebuchbinderei, Nehren 


\section{"Nous aurons entendu cela." Temporalität und Modalität - zur Dynamik der Kategorienorganisation beim futur antérieur}

\section{Tempus und Prozesse der Grammatikalisierung - einige Vorüberlegungen}

Die Verbindung von Tempus und Grammatikalisierung ist in der Sprachwissenschaft im Bereich der Grammatikalisierungsforschung keineswegs neu. ${ }^{1}$ Die Schwerpunkte liegen hier bei der Emergenz von Tempusformen und bei der Untersuchung von Pfaden der Grammatikalisierung, die die Funktionsveränderungen von Tempusformen als Kontinuum von Entwicklungsstadien beschreiben. Beide Aspekte sind am Beispiel des französischen Futurs mehrfach diskutiert worden, das als grammatische Kategorie aufgrund seiner 'Labilität' für diachronische Untersuchungen besonders ergiebig scheint und reiches Material für die Diskussion von Prozessen der Grammatikalisierung, gerade auch der Verflechtungen von Grammatikalisierung und Reanalyse, bietet. $^{2}$

Der vorliegende Beitrag ordnet sich zwar in die Tradition der Verknüpfung von Tempus und Grammatikalisierung ein, wählt jedoch eine synchronische Perspektive, die auch eine Verschiebung der Schwerpunkte impliziert - nicht mehr die Entwicklung der Form, sondern ihre Funktionen in der Synchronie sollen gestützt auf Modelle der Grammatikalisierung beleuchtet werden. Eine solche Beschränkung auf die Synchronie erscheint legitim, da Prozesse der Grammatikalisierung zunehmend als Phänomene beschrieben werden, bei denen sich die diachronische und die synchronische Perspektive, die an Boden gewonnen hat, synoptisch ergänzen. Synchronisch angelegte Ansätze erfassen dabei grammatische Kategorien als im Wandel befindliche, unfeste Größen, in denen ältere Funktionen und neuere, sich entwickelnde Werte kopräsent sind. Der sich graduell vollziehende Bedeutungs- und Funktionswandel als Dynamik einer Kategorie wird dabei nicht mehr diachronisch als Sequenz von Entwicklungsstadien etabliert, sondern entfaltet sich synchronisch als Vielfalt der semantisch-pragmatischen Verwendungstypen einer Form. Diese Vielfalt der Funktionstypen ist nun bei einer französischen Tempusform, dem futur antérieur, in besonderem Maße gegeben, das damit exemplarisch die Dynamik der Kategorienorganisation auch in der Synchronie zu illustrieren vermag. Ein solcher Blick auf eine Form mit vielen Funktionen beinhaltet ein doppeltes Erkenntnisinteresse. Zum einen kann das Raster der Grammatikalisierungstheorie helfen, das semantisch-pragmatische Potential des futur antérieur im heutigen Französisch zu erklären und die Verwendungen dieser unfesten Kategorie so zu systematisieren. ${ }^{3}$ Umgekehrt verspricht eine solche Untersuchung auch Aufschlüsse über die Aussa-

1 Für Anregungen, Kommentare und konstruktive Kritik danke ich Franz Lebsanft und Peter Koch sowie Jurgen Lang und Ingrid Neumann-Holzschuh. Mein Dank gilt ferner Claire Chesnais, die sich als Muttersprachlerin in Sachen futur antérieur von mir befragen ließ. Vgl. Fleischman (1982); Schwegler (1990); Roberts (1993); Bybee/Pagliuca/Perkins (1991) und (1994).

Vgl. Campbell (1991: 285). 
gekraft prominenter Konzepte des Sprachwandels. Am Beispiel des futur antérieur und seiner scheinbar unverbundenen temporalen und modalen Werte sollen Mechanismen und Modelle des Bedeutungs- und Funktionswandels besprochen und auf ihre Erklärungskraft geprüft werden. Im Zentrum stehen dabei Modelle, die metaphorische und metonymische Prozesse als Movens der Grammatikalisierung analysieren, und Ansätze, die die pragmatische Dimension des Sprachwandels vertiefen.

Im Hinblick auf den gewählten pragmatischen Schwerpunkt ergibt sich übrigens ein weiterer Berührungspunkt zwischen Tempusforschung und Grammatikalisierungstheorie, da beide Zweige vermehrt eine Öffnung zur Pragmatik vollziehen. In der Tempusforschung gilt die Aufmerksamkeit immer mehr den Tempusverwendungen im Text und dem pragmatischen Potential der Futura. ${ }^{4}$ Diese Hinwendung zur pragmatisch-textuellen Komponente entspricht damit einer Tendenz in der Grammatikalisierungsforschung, die vor allem in frühen, durch Bedeutungswandel geprägten Stadien der Grammatikalisierung wesentlich pragmatische Kräfte am Werke sieht, so daß beide Richtungen sich hier ergänzen. ${ }^{5}$

Der synchronische Schwerpunkt bedingt, daß eine Rekonstruktion der semantischen und funktionalen Entwicklung des futur antérieur nicht untemommen wird, auch wenn die vorliegende Untersuchung vereinzelt in die Diachronie ausgreift. Nur eine Ausweitung der Untersuchung auf Corpora unterschiedlicher Entwicklungsstadien des Französischen könnte Gewichtsverlagerungen innerhalb der Funktionstypen des futur antérieur zuverlässig dokumentieren und damit einen möglichen Kategorienwechsel der Tempusform erfassen. Rückschlüsse auf die Entwicklungsgeschichte des futur antérieur, die allein von einer synchronen Polysemiestruktur ausgehen würden, wären dagegen mit erheblichen Unsicherheiten behaftet. ${ }^{6}$

Im folgenden werden zunächst die für meinen Ansatz relevanten Modelle der Grammatikalisierung kurz präsentiert, bevor ich die temporalen und modalen Funktionen des futur antérieur vorstelle, deren Relationen zueinander dann die Dynamik der Kategorienorganisation exemplarisch illustrieren sollen.

\section{Prozesse der Grammatikalisierung - theoretische Prämissen und Modelle}

Den Prozeß der Grammatikalisierung fasse ich mit Traugott/Heine (1991a) ${ }^{7}$ als graduellen Prozeß der Kategorienorganisation und der Kategorienkodierung auf, der diachronisch oder synchronisch untersucht werden kann. Die diachronische Betrachtung beschreibt aus einer "source and pathway"-Perspektive die Pfade der Kategorienentwicklung und untersucht Veränderungen

\footnotetext{
Vgl. Fleischman (1983: bes. 186ff., $201 \mathrm{ff}$.) zum pragmatischen Potential der Tempusformen.

Traugott/Hopper (1993: 68, 207): "the meaning changes are initially pragmatic and associative and arise in the context of the flow of the speech".

6 Dies versucht Traugott (1990: 499f., 513); vgl. hierzu kritisch Laca (1996: 22f.).

7 Traugott/Heine (1991a: 1): "Grammaticalization is the linguistic process, both through time and synchronically, of organization of categories and of coding". Abraham (1992/93: 8): " Grammatikalisierung ist der linguistische, diachrone ebenso wie synchrone Prozeß der Kategorienorganisation und seiner Kodierung". Vgl. auch Lehmann (1985: 303); Traugott/Hopper (1993: 2).
} 
der "grams" im Verlauf der Kategorienentwicklung, wie etwa den Verlust an Autonomie und den Wandel von Bedeutungen und Funktionen. ${ }^{8}$ In dieser diachronischen Perspektive wird etwa verfolgt, wie sich Futura als Ausdruck epistemischer Modalität aus agensorientierten Modalitäten des Könnens und der Verpflichtung entwickeln und dann in einem nächsten Stadium weitere modale Werte erwerben. ${ }^{9}$ Ergänzend dazu werden sprachliche Kategorien aus synchronischem Blickwinkel als "fluid patterns of language use" aufgefaßt, die in ihrer Semantik und ihrem pragmatischen Potential die Spuren prozeßhafter sprachlicher Veränderungen tragen. ${ }^{10}$ Synchronisch manifestiert sich die Entwicklung neuer Funktionen als Koexistenz älterer und neuerer semantisch-pragmatischer Werte in einer Kategorie. Die graduelle Natur des Grammatikalisierungsprozesses bedingt dieses Zwischenstadium des "split", ${ }^{11}$ da eine sprachliche Form im Prozeß der Veränderung alte Verwendungen beibehält, während sich bereits neuere entwickeln. ${ }^{12}$ Die ältere Bedeutung bzw. Funktion kann dabei lange Zeit weiterbestehen, so daß sich stabile Polysemien ergeben. ${ }^{13}$ Der von mir gewählte synchronische Ansatz, der das futur antérieur als eine 'unfeste' Kategorie mit kopräsenten temporalen und modalen Funktionen darstellt, konzentriert sich auf diese semantisch-pragmatische Seite der Kategorienorganisation als einen wichtigen Teilaspekt der Grammatikalisierung. ${ }^{14}$ Im 'Standbild' des Französischen der Gegenwart wird damit eine Polysemie der Kategorie futur antérieur beschrieben, in der sich der Wandel von Bedeutung und Funktion manifestiert. Diese Fokussierung auf die semantisch-pragmatische Kategorienorganisation versteht sich auch als Ergänzung zu Ansätzen, die dem Bedeutungswandel bei der Behandlung von Grammatikalisierungsprozessen eine eher marginale Position zuweisen. ${ }^{15}$ Die dargelegten Sichtweisen der Kategorienorganisation stellen damit zwei Seiten einer Medaille dar. Der Wandel von Bedeutung und Funktion manifestiert sich diachronisch als unidirektionelle Entwicklung und synchronisch als Netzwerk korrelierter Prozesse, ${ }^{16}$ so wie sich ja auch in der Historischen Semantik Bedeutungswandel in der Diachronie und Polysemie in der Synchronie als zwei Betrachtungsweisen ein und desselben Phänomens ergeben. ${ }^{17}$

Aus dieser Perspektive stellen sprachliche Kategorien unfeste Größen dar, die in ihrer Dynamik von der Rede im Sinne Humboldts geformt werden. ${ }^{18}$ Diese Dynamik einer Kopräsenz von

Lehmann (1985: 306, 310) und Lehmann (1995: 11-14); Heine/Claudi/Hünnemeyer (1991a: 4).

9 Bybee/Pagliuca/Perkins (1991: 23ff., 25-29); vgl. auch Bybee/Pagliuca (1985: 63-66, 69) und Heine/Claudi/Hunnemeyer (1991 a: 176).

10 Traugott/Heine (1991a: 6). Vgl, auch Lehmann (1985: 303), dem zufolge synchronisch betrachtet Grammatikalisierung ein Prinzip meint "according to which subcategories of a given grammatical category may be ordered"; vgl. auch Abraham (1992/93: 9).

11 Heine/Claudi/Hünemeyer (1991b: 162, 168) und (1991 a: 67, 74); Traugott/Hopper (1993: 120, 124).

12 Heine/Claudi/Hünemeyer (1991b: 155ff., 162) und (1991 a: 65ff., 70-72); Hopper (1991: 22f., 28f., 30f.); Traugott/Hopper (1993: 120)

13 Zur langen Dauer der Koexistenz vgl. Traugott (1989: 33f); Heine/Claudi/Hünnemeyer (1991b: 166, 172, 180).

14 Im Zentrum steht also die semantisch-pragmatische Kategorienorganisation und weniger die Kodierung der Kategorie, die in diachronischen Ansătzen meist dominiert, wie auch die Definition Lehmanns verdeutlicht (1985: 303): "Under the diachronic aspect, grammaticalization is a process which turns lexemes into grammatical formatives and makes grammatical formatives still more grammatical [...]".

15 Vgl. z.B. Lehmann (1995: 121-160).

16 Traugott/Heine (1991a: 6). Vgl. auch Abraham (1992/93: 9) und Lehmann (1985: 303).

17 Vgl. hierzu auch Koch (1995: 28).

18 Lichtenberk (1991: 37, 67); Taylor (1989: 91); Bybee/Pagliuca/Perkins (1991: 18) 
semantisch-pragmatischen Werten dokumentiert den Prozeß der Grammatikalisierung als kontinuierliche Bewegung und Emergenz von Strukturen und Funktionen, die angetrieben ist von den Kräften der Interaktion zwischen den Sprechern, ihren Bedürfnissen und ihrer Kreativität einerseits und dem Funktionspotential des bestehenden Formeninventars andererseits. ${ }^{19}$ Bei der Untersuchung des futur antérieur gilt es nun zu prüfen, inwiefern die verschiedenen Funktionstypen der Form ein solches Zwischenstadium der Entwicklung abbilden könnten. Der Versuch, synchronisch ein Netzwerk von Entwicklungslinien zu eruieren, fokussiert einen Aspekt der Grammatikalisierung, nämlich die Dynamik der Kategorienorganisation als Wandel von Bedeutung und Funktion. Die unter diesen Vorzeichen unternommene Verknüpfung von Tempus und Grammatikalisierung berührt nun die Reanalyse als an der Grammatikalisierung beteiligten semantisch-syntaktischen Prozeß zunächst nicht, sondem konzentriert sich ganz auf semantischpragmatische Modelle der Grammatikalisierung. Bei der Erarbeitung der Erklärungsmuster für die vielfältigen Funktionen des futur antérieur ergibt sich allerdings auf Modellebene eine Analogie zur Reanalyse. In einer weitgefaßten Definition meint Reanalyse einen Fall von metonymischem Bedeutungswandel, bei dem sich durch das Inferenzpotential eines Ausdrucks die syntagmatischen Relationen innerhalb der Einheiten dieses Ausdrucks verändern. ${ }^{20}$ Der Funktionswandel kommt also zustande durch eine modifizierte syntagmatische Relationierung der Elemente, die ausdrucksseitig intakt bleiben - verändert wird also nur die syntaktisch-semantische Konfiguration. Auf diesen Mechanismus einer 'Konfigurationsänderung', die die Elemente als solche intakt beläßt, werde ich bei der Deutung der Funktionsvielfalt des futur antérieur noch zurückkommen.

Für meine Vorgehensweise ist nun noch ein weiterer Grundsatz neuerer Beiträge zur Reanalyse prägend. Wie die Grammatikalisierung allgemein wird auch die Reanalyse vermehrt aus der diachronischen Blickrichtung herausgelöst. ${ }^{21}$ Während diachronische Betrachtungen diesen Prozeß als historische Entwicklung und Kategorienwechsel erst in einer späten morphosyntaktischen Gerinnungsphase erfassen, gilt das Interesse der synchronischen Betrachtung dem Aufspüren semantisch-pragmatischer Vorstufen, die einen Kategorienwechsel in statu nascendi anzeigen und motivieren. ${ }^{22}$ Im Zentrum des Interesses stehen damit sich anbahnende Veränderungen in der semantischen Konfiguration einer Kategorie ${ }^{23}$ und die konzeptuell-kognitiven Motive, die

19 Vgl. auch Heine/Claudi/Hünemeyer (1991 a: 248-252).

20 Die gegebene Definition übernimmt eine in der durch den vorliegenden Band dokumentierten Sektion des Jenaer Romanistentags 1997 von Ulrich Detges, Georg A. Kaiser, Elisabeth Stark, Richard Waltereit und mir vorgeschlagene 'Arbeitsdefinition'. Langacker (1977: 58, 62f., 64) vertritt einen weiten Begriff von Reanalyse als Wandel von Bedeutung und Funktion ohne ausdrucksseitige Veränderung, der auch Falle von Kategorienänderung durch Bedeutungswandel einschließen konnte (vgl. ebd. 119f. zur semantic shift). In einer engeren Definition wie bei Abraham (1992/93: 14) und Traugott/Hopper (1993: 40-42) dagegen wird Reanalyse mit einer Veranderung von morphologischen oder syntaktischen Konstituentengrenzen verknupft. Engere Definitionen korrelieren die Veränderung der syntagmatischen Relationierung also einschrănkend mit einer Veränderung der Konstituentenstruktur. Die in der Sektion besprochene 'Enge' bzw. 'Weite' der Begriffsdefinition variiert auch innerhalb der einzelnen Forschungsansätze. Zum Verhältnis von Reanalyse und Grammatikalisierung vgl. Heine/Claudi/Hünemeyer (1991a: 215-217, 219f.); Abraham (1992/93: 13-14); Traugot//Heine (1993: 48-50).

Abraham (1992/93: 7f., 8-10).

22 Abraham (1992/93: 14f., 22); Schrodt (1993: 262).

23 Nach Langacker (1977: 68, 79f.) wăre dies die reformulation im Unterschied zur mit der Veränderung der Morphemgrenzen befaßten resegmentation. 
diesen Bedeutungswandel motivieren könnten. ${ }^{24}$ Dieser Gesichtspunkt des Reanalysemodells ist auch ein Leitfaden für die Darlegung des semantisch-pragmatischen Potentials des futur antérieur. ${ }^{25}$ Die Polyfunktionalität des futur antérieur wird als Momentaufnahme einer Entwicklung begriffen, deren Richtung sich im "split"-Stadium bereits andeuten könnte - zentral ist also der auch methodisch zu reflektierende Versuch, Spuren einer sich andeutenden Veränderung der Kategorienorganisation zu entdecken.

Die im Prozeß der Grammatikalisierung wirkenden Kräfte sind in der Forschung in unterschiedlichen Modellen strukturiert worden. Die Kopräsenz der Verwendungstypen des futur antérieur soll vor dem Hintergrund eines von Traugott (1982) entwickelten Modells dargelegt werden, das frühe Stadien der Grammatikalisierung über eine pragmatische Aufladung von Kategorien erklärt, die zunehmend textuelle und expressive Funktionen übernehmen. Nach Traugott (1982) erfahren sprachliche Kategorien im Prozeß der Grammatikalisierung eine semantischpragmatische Verschiebung, die über drei funktional-semantische Ebenen der Sprache verläuft. ${ }^{26}$ Eine Form hat zunächst propositionalen Gehalt, erfült dann textuelle Funktionen und wird schließlich im expressiven Bereich Ausdruck einer Sprecherhaltung gegenüber der Proposition, so daß die versprachlichten Sachverhalte gespiegelt in einer "speaker's world of belief" erscheinen. ${ }^{27}$ Die Übergänge vom propositionalen zum textuellen und expressiven Bereich werden als metaphorische Prozesse aufgefaßt, bei denen abstrakte Bedeutungen durch weniger abstrakte Konzepte mit dem Ziel einer besseren kognitiven Repräsentation versprachlicht werden. ${ }^{28}$ In diesem Zusammenhang sollte präzisiert werden, daß Metaphern zwar eine konzeptuelle Basis haben, aber sprachliche Verfahren darstellen. Nach Koch $(1994,1995,1996)^{29}$ basieren Metaphern auf einer Similarität auf Designatsebene, die sich zwischen zwei Konzepten aus distanten Bereichen bzw. Frames ergibt, wenn in einem konzeptuellen Sprung ein Herkunftskonzept auf ein Zielkonzept projeziert wird.

Während im Traugottschen System ${ }^{30}$ die metaphorischen Übergänge als miteinander korrelierte zeitlich aufeinanderfolgende Tendenzen verstanden werden, übernehme ich das Modell in einer modifizierten Form. Die drei Phasen werden nicht mehr als feste Sequenz von Entwicklungsstadien aufgefaßt, sondern als verknüpfte Werte, die in einer polysemen Kategorie in einem Entwicklungsstadium gegeben sind. ${ }^{31}$ Die Hypothese einer Kopräsenz der drei Bereiche stützt

24 Aus der kognitiven Sicht von Heine/Claudi/Hunnemeyer (1991b: 168) erscheint Reanalyse als "cases where conceptual manipulation [...] causes a linguistic structure to be treated as some alternative structure".

Vgl. auch Schrott (1997: 209-224, 294-332).

Traugott (1982: 247f.).

Traugott/Konig (1991: 192f.); Traugott (1982: 253, 255f.)

Traugott/Hopper (1993: 77).

Vgl. Koch (1994: 210f., 213f.); Koch (1995: 39) und (1996: 233).

Traugott/König (1991: 208f.); Traugott (1989: 34f.) und (1990: 499f.). Das Modell Traugotts wird im Hinblick auf das gewăhlte Untersuchungsobjekt des futur antérieur leicht vereinfacht wiedergegeben.

31 Das Postulat der zeitlichen Abfolge ist auch in der Forschung nicht unumstritten. Nach Herring (1991: 255, 279) und Heine/Claudi/Hunnemeyer (1991 a: 243) können grammatische Elemente von der propositionalen oder von der diskurspragmatischen Ebene kommen, beide Prozesse sind unabhängig voneinander und kơnnen auch frei interagieren. 
sich auf meine Untersuchungen zu futur simple und futur périphrastique, die zeigen, daß die Futura im heutigen Französisch ebenfalls sowohl temporal-situierende als auch textuelle und expressive Funktion haben. ${ }^{32}$

Diese pragmatisch motivierte Grammatikalisierungstendenz kann nun besonders deutlich bei der Entwicklung von Tempusformen beobachtet werden. Die zeitliche Semantik der Tempora, die propositional eine Situierung von Sachverhalten relativ zum ego-hic-nunc leisten, wird metaphorisch auf die textuelle und expressive Funktionsebene übertragen, ${ }^{33}$ wobei die zeitliche Ferne als Sprecherdistanz, die zeitliche Nähe als Aktualität und Sprecherpräsenz erscheinen. Die Tempussemantik wird also von der Sprechsituation auf eine Textsituation übertragen, in der das Tempus dann innerhalb der Textwelt situierend wirkt. In textueller Funktion strukturieren Tempora durch Fokussierungen den Text und markieren die Quellen des propositional vermittelten Wissens, wobei die Sprecherdistanz unsicheres Wissen, die Sprecherpräsenz sicheres Wissen impliziert. ${ }^{34}$ Diese textuelle Funktion, in der sich bereits der Sprecher als deutendes Subjekt manifestiert, geht dann über in die expressive Textfunktion der Sprecherwelt, bei der das Tempus dann Sachverhalte in der Welt der Überzeugungen des Sprechers situiert ${ }^{35}$ und so die Sprecherinstanz im Text konstituiert. ${ }^{36}$ Die Diskussion von Textbeispielen soll zeigen, ob dieses Modell einer Entwicklung vom propositionalen Gehalt hin zu pragmatisch intensivierten textuellen und expressiven Funktionen eine Systematisierung und Vernetzung der Funktionstypen des futur antérieur zu leisten vermag.

Neben dem metaphorisch motivierten Modell Traugotts greife ich noch auf das Modell eines metonymischen Mechanismus des Bedeutungswandels zurück, die "context-induced reinterpretation" ${ }^{37}$ Wie Metaphern so funktionieren auch Metonymien auf konzeptueller Basis und beruhen auf einer Kontiguität von Elementen, die einem Bereich bzw. Frame angehören. ${ }^{38}$ Wenn eine Form häufig in Kontexten auftritt, in denen eine bestimmte Inferenz gegeben ist, dann besteht Kontiguität zwischen der Form und der Inferenz, die den Status einer kontextuell bedingten konversationellen Implikatur hat. ${ }^{39}$ Diese Kontiguität von Form und konversationeller Implikatur bewirkt, daß beide Größen konversationell verknüpt werden, so daß die Implikatur allmählich in die Semantik der Form übergeht und als Teil der Bedeutung konventionalisiert wird. Die so integrierten Inferenzen sind pragmatischer Natur und bewirken daher eine pragmatische Aufladung der Bedeutung. ${ }^{40}$ Diese Aufladung geht nun - wie schon das metaphorische Konzept -

Vgl. dazu Schrott (1997: 117-141).

Fleischman (1989: 2f., 5f.). In einem fruheren Aufsatz (1983: 185f., 190f.) vertritt Fleischman noch die These, daß Tempusformen sich aus pragmatischen Quellen heraus zu Tempora entwickeln. Nach Fleischman sind perfect-Formen durch die "present relevance" pragmatisch stärker markiert als Tempora und verlieren diese Markierung im Laufe ihrer Entwicklung zu reinen Tempora; vgl. hierzu auch Traugott (1982: 56).

Fleischman (1989: 25f., 37f.).

Traugott/König (1991: 209); vgl. auch Traugott (1990: 501); Heine/Claudi/Hünnemeyer (1991 a: 179-181).

Lakoff (1970: 838-84 1); Mair (1992: 45, 100, 113-115).

Heine/Claudi/Hünnemeyer (1991b: 45ff., 48ff., 161-167, 171, 181); Traugott/König (1991: 210); Traugott/Hop-

per (1993: 72ff., 75); Anttila (1972: 14lf.).

Koch (1995: 40) und (1996: 236).

Nach Taylor (1989: 91) funktionieren Inferenzen uber ein skript, das Sachverhalte innerhalb von Handlungsschemata miteinander verbindet und so auch temporale und kausale Werte verknupft.

Traugott/Heine (1993: 81); Traugott/König (1991: 192f., 211); Heine/Claudi/Hünnemeyer (1991a: 61-63). 
mit einer stärkeren Zentrierung der Form auf die Sprecherinstanz einher, so daß solcherart pragmatisch 'gesättigte' Tempora als Teil ihrer Semantik Sprecherillokutionen versprachlichen. ${ }^{41}$

Die dargelegten metaphorischen und metonymischen Prozesse werden nun in ihrer Relation zueinander unterschiedlich beurteilt. So werden Metaphern und Metonymien einerseits mit unterschiedlichen Prozessen der Grammatikalisienung verbunden, ${ }^{42}$ andererseits als Teile eines umfassenderen Mechanismus verstanden. Gegenüber dem Konzept der getrennten Aufgabenbereiche gewinnt ein Modell an Boden, das Metapher und Metonymie als ineinandergreifende Prozesse deutet. ${ }^{43}$ Eine solche Interaktion ist gegeben, wenn sich metaphorische Transfers durch den metonymischen Prozeß kontextbedingter Deutungen allmählich im Sprachgebrauch einbürgern. Der an sich diskrete metaphorische Transfer zwischen zwei distanten Konzepten verläuft nämlich über ein Zwischenstadium, das Stadium des "split", in dem beide Konzepte metonymisch koexistieren. In einer Form, die sich etwa von der textuellen hin zu einer expressiven Bedeutung entwickelt, sind beide Bedeutungen kopräsent und die jeweiligen, in Kontiguität zur Form stehenden Kontexte bestimmen, welche Deutung als die relevanteste erscheint. Diese Deutung wird dann im Sinne der "context-induced reinterpretation" immer mehr in die Semantik der Form integriert. Die verschiedenen Funktionen des futur antérieur und ihre Verflechtungen werden also auch auf die Mechanismen von Metapher und Metonymie und ihre Relation zueinander zu hinterfragen sein.

\section{Die Verwendungstypen des futur antérieur}

Die besprochenen Modelle der Bedeutungsveränderung und ihre Interaktion sollen nun anhand der Funktionen des futur antérieur diskutiert werden, die eine temporal-futurische Verwendung und modale Gebrauchstypen umfassen. Da das semantisch-pragmatische Potential dieser Funktionstypen sich nur bei der Verwendung in konkreten Kontexten manifestiert, werden die Verwendungen des futur antérieur im folgenden anhand von Textbeispielen diskutiert. ${ }^{44}$

\subsection{Das temporal-futurische futur antérieur}

In der temporal-futurischen Verwendung versprachlicht das futur antérieur Sachverhalte, die in einer zukünftigen Referenzsituation bereits abgeschlossen sind und deren Realisierung damit vorzeitig zu dieser futurischen Referenzsituation zu lokalisieren ist. In den folgenden Beispielen

41 Bybee/Pagliuca/Perkins (1991: 23-29); vgl. auch Bybee/Pagliuca (1985: 63-66, 69); Heine/Claudi/Hunnemeyer (1991 a: 176); Traugott (1989: 31-33, 43); Traugott (1989: 49f).

42 Vgl. hierzu etwa die Ausfuhrungen bei Traugott/Konig (1991: 213).

43 Heine/Claudi/Hunnemeyer (1991b: 155-157, 162) und (1991 a: 65-67, 70-72); vgl. auch Traugott/Hopper (1993: 120).

44 Vgl. Laca (1996: 21). 
ist dieser zeitliche terminus ante quem explizit gegeben: ${ }^{45}$

(1) Un Européen sur dix connaît Lyon par le tunnel de Fourvière: belle image! Et quel automobiliste français n'a pas été bloqué au moins une fois dans le tunnel de Fourvière: belle image! Alors c'est simple: fin décembre 1991, nous aurons fait sauter au $3 / 4$ ce fameux bouchon ... et fin 92 en totalité! Parole de Lyonnais. Vive les vacances $92,93,94 \ldots$ et merci Lyon! [NO 1399, S. 10]

(2) Tony Blair, travailliste et séducteur.

C'est, en termes de showbiz, ce qu'on appelle mettre une salle dans sa poche. Au rythme qui est le sien, Tony Blair aura bientôt mis tout le Labour - les syndicats comme les militants - dans son mouchoir de soie. En attendant que la fièvre "blairiste" gagne toute l'Angleterre ... [NO 1591, S.7]

Das futur antérieur versprachlicht hier, daß ein Sachverhalt vor einer futurischen Referenzsituation abgeschlossen sein wird, so daß in dieser Situation dann ein bestimmter Tatbestand gegeben sein wird. So wird in (1) die Beseitigung des Engpasses zum (damals noch) futurischen Zeitpunkt "fin décembre 1991" zum größten Teil beendet sein, und in (2) wird Tony Blair - wir schreiben den Mai 1995 - in Kürze die Labour Party in die Tasche gesteckt haben.

Das beim futur antérieur gegebene Konzept der Vorzeitigkeit in der Zukunft enthält zwar den futurischen Referenzpunkt als terminus ante quem, legt aber die Lokalisierung des abgeschlossenen Sachverhalts in Relation zur deiktischen origo nicht fest. ${ }^{46}$ Entscheidend ist, daß der Sachverhalt abgeschlossen ist und daß in der futurischen Referenzsituation ein aus dem abgeschlossenen Sachverhalt resultierender Zustand gegeben ist. Geht man vom prototypischen Modell einer Situation mit den Verlaufsphasen Vorbereitungsphase, Transition und Folgephase aus, ${ }^{47}$ dann versprachlicht das futur antérieur einen Sachverhalt, der eine Transition beinhaltet und in eine Folgephase mündet. Dabei ist das futur antérieur durch eine Fokussierung der futurischen Folgephase charakterisiert, während der nur vage situierte abgeschlossene Sachverhalt weniger akzentuiert erscheint ${ }^{48} \mathrm{Da}$ das futur antérieur den versprachlichten Sachverhalten damit eine temporale Kontur aufprägt, wird die Form in der Forschung meist nicht als deiktisch situierendes Tempus, sondern als Aspekt eingeordnet, ${ }^{49}$ und zwar als aspect (de $l^{\prime}$ )accompli, bei dem aus einer abgeschlossenen Handlung ein resultativer Zustand entsteht. ${ }^{30}$ Diese Relation zwischen einem Sachverhalt und einem Folgezustand wird auch durch das Konzept der "present relevance" erfaßt, die einen Sachverhalt und einen in einer Referenzsituation gegebenen Zustand miteinander verbindet. ${ }^{51}$ Im Falle des futur antérieur erscheint der Zustand retrospektiv als Folgephase aus einem abgeschlossenen Sachverhalt, der Sachverhalt ist damit in der futurischen Referenz-

Diese Darlegung findet sich bei Damourette/Pichon (1936: 455); Imbs (1960: 109ff., 81ff.); Togeby (1982: 421); Comrie (1981: 26) und (1985: 7lff., 126f.). Zum futur antérieur vgl. auch Wilmet (1976: 41-60, bes. $44 f ., 57-$ 60).

46 Damourette/Pichon (1936: 455); Reichenbach (1947: 296f.); Togeby (1982: 421); Helland (1994: 38-40).

47 Vet (1980: 62-72, bes. 68ff.) und (1991: 14-16); Moens/Steedman (1988: 17f.).

48 Diese Einschătzung findet sich auch bei Vet (1980: 89); Heger (1963: 112); Wunderli (1989: 77f); Smith (1991: 274); Bybee/Pagliuca/Perkins (1994: 63-68).

49 Zur Definition des Aspekts vgl. Heger (1963: 22ff.); Comrie (1976: 5); Coseriu (1980: 14f.); Vet (1980: $45,77-$ 80); Fleischman (1982: 11 ); Maslov (1985: 3f.).

so Imbs (1960: 109f.); Vet (1980: 79, 88f.); Wunderli (1989: 77f.); Wilmet (1976: 44, 145-147).

51 Comrie (1976: 64f.); Vet (1980: 71); Fleischman (1982: 18f., 95-98, bes. 96f.) und (1983: 191ff.) 
situation noch pars pro toto als Resultat gegeben. ${ }^{52}$ Formen, die wie das futur antérieur durch "present relevance" markiert sind, werden bei Kortmann $(1991)^{\mathbf{5 3}}$ durch eine eigene Kategorie, den "Anterior", erfaßt. Diese Einstufung trägt der Tatsache Rechnung, daß das futur antérieur in sich Eigenschaften eines nicht-deiktischen Aspektes und einer deiktischen Tempusform vereinigt. Im Fall des futur antérieur kommt zur aspektuellen Markierung eine deiktische Komponente, denn wenn auch der abgeschlossene Sachverhalt bezüglich der Referenzsituation unbestimmt ist, so ist die Folgephase doch nachzeitig zur Sprechsituation situiert. Das futur antérieur wird also im folgenden als Anterior-Form eingeordnet, bei der ein vorzeitiger Sachverhalt in Relation zu einem späteren Zustand gesetzt wird.

\subsection{Der modale Funktionstyp des futur antérieur de probabilité}

Nach der Behandlung des temporal-futurischen Wertes soll nun noch auf weitere Verwendungen eingegangen werden, die modalen Wert haben. Dabei gehe ich von der Arbeitshypothese aus, daß diese scheinbar divergenten Gebrauchstypen auf eine einheitliche Basis zurückgeführt werden können. ${ }^{54}$ Im folgenden werden sprachliche Elemente als Ausdruck von Modalität aufgefaßt, wenn sie die Präsenz des Sprechers in der Sprache signalisieren und eine Haltung des Sprechers gegenüber der geäußerten Proposition ausdrücken. ${ }^{\text {ss }}$ Der modale Gehalt der im vorangegangenen Abschnitt beschriebenen temporalen Verwendungen des futur antérieur besteht nun in der Haltung des Sprechers, der dem futurischen resultativen Zustand eine subjektive Gewißheit ${ }^{56}$ zu- $^{-}$ schreibt, den künftigen Sachverhalt in sein "univers de croyance" ${ }^{57}$ integriert und als Garant eine kommunikative Regreßpflicht für diese Vorhersage übernimmt. ${ }^{58}$ Dieser Grad der subjektiven Sprechergewißheit konstituiert die epistemische Modalität des futur antérieur, die die modale 'Grundierung' der temporalen Verwendungen bildet, ${ }^{59}$ so daß das futur antérieur durch eine Du-

Kleiber/Berthonneau (1993: 56ff., 66ff.). Ein verwandter Ansatz findet sich bei Moens/Steedman (1988: 16), die die nicht-temporale Tempussemantik mit dem Begriff der Kontingenz charakterisieren, der von den Verfassern allgemein als eine semantische Verbindung definiert wird, die nicht rein temporaler Natur ist. Kontingenz erscheint als "a term related, but not identical to a notion like causality".

53 Kortmann (1991: 14f., 17f., 20f.). Die Kategorie "anterior" kann past perfect, present perfect und future perfect umfassen. Vgl. auch Bybee/Pagliuca/Perkins (1994: 51-105), die diese Kategorie unter dem Etikett "resultative" abhandeln (63-68) und auch verwandte temporal-aspektuelle Werte besprechen.

54 Coseriu (1976: 46ff.); Comrie (1985: 18f., 24); Dahl (1985: 9f.); Taylor (1989: 18ff., 59-65, 173ff.).

55 Herslund (1989a: 7f., 15): "Modality, as a semantic cover term, is then manifested in different linguistic categories and syntactic processes which all have in common that they signal the speaker's attitude towards the propositional content of what he is saying: it is not so much what he is saying, it is the way he is saying it" (hier 1989a: 15). Vgl. auch Fleischman (1982: 13f).

56 Heger ( $\left.{ }^{2} 1976: 280 \mathrm{ff}\right)$.

57 Martin (1981: 84); Martin (1983: 126ff., 131ff.) und (1987: 10, 15ff., 20, 36ff., 38f.): "la vérité de toute phrase déclarative étant une vérité subjectivement assumé par un locuteur, une vérité prise en charge, le vrai 'objectif n'a pas de réalité linguistique" (hier 1987: 38).

59 Vgl. auch Damourette/Pichon (1936: 385ff.); Palmer (1986: 51); Comrie (1989: 53f.). 
plizität von Temporalität und Modalität gekennzeichnet ist. ${ }^{60}$

Diese epistemische Modalität als Kehrseite der Futurität bildet nun keine modale Verwendung, die sich vom temporal-futurischen Wert abgrenzt oder gar in Widerspruch zur temporalen Verwendung steht. Eine solche Diskrepanz zwischen temporal-futurischem und modalem Wert eröffnet sich jedoch beim Verwendungstyp des futur de probabilité. In der Funktion eines futur de probabilité referiert das futur antérieur nämlich auf Sachverhalte mit vergangener Zeitreferenz und formuliert diese Sachverhalte als Hypothesen zu einem bereits abgeschlossenen Sachverhalt. ${ }^{61} \mathrm{Im}$ folgenden Beispiel stellt ein Journalist Vermutungen über den Tathergang eines Doppelmordes an:

(3) Les déclarations de Maria-Antonia sont confuses. Elle a peut-être avoué aux Cluzel qu'elle avait tué Patricia. Ils ont dû avoir une expression horrifiée, s'approcher d'elle en levant les bras au ciel, ce qu'elle aura pris pour un geste de menace. Plus tard, elle s'est laissé conduire au commissariat, le regard absent. [NO 1527, S.13]

Beim Versuch, die Hintergründe eines Doppelmordes zu rekonstruieren, stellt der Verfasser die Hypothese auf, die Täterin habe sich bedroht gefühlt und daher das Ehepaar Cluzel ermordet, er läßt sich für diese Vermutung aber nur bedingt in die kommunikative Regreßpflicht nehmen. ${ }^{62}$

Der modale Wert der Hypothese kann durch eine metaphorische Umdeutung der Futurität erklärt werden, die kontextuell ausgelöst wird. ${ }^{63}$ Referiert das futur antérieur auf nicht-futurische Sachverhalte, dann widerspricht diese Zeitreferenz der futurischen Semantik der Formen und löst eine Neubewertung der temporalen Semantik aus. Basis für diese metaphorische Umdeutung ist, daß temporal-futurische Sachverhalte in der Sprechsituation noch nicht verifizierbar sind und daher durch die Futurität auch das Kriterium der verschobenen Verifizierbarkeit beinhalten. Verbindet man diese Interpretation mit den einleitend referierten Modellen der Grammatikalisierung, dann sind hier Metapher und Metonymie verknüpft. Denn zur metaphorischen Umdeutung der Futurität hin zu einer Nicht-Verifizierbarkeit kommt noch die metonymische Kontiguität von futur antérieur und nicht-futurischem Kontext, die diese Umdeutung auslöst - Metapher und Metonymie funktionieren hier also in enger Verschränkung. Durch diesen metaphorischen Übergang verliert das futur antérieur die propositionale Funktion der futurischen Situierung und gewinnt einen textuell-expressiven Wert. Die durch das futur de probabilité ausgedrückten Sachverhalte werden in der Textwelt als Hypothesen versprachlicht und indizieren eine Sprecherdistanz: Der Sprecher ist zwar Urheber der Hypothese, er übernimmt aber nur eine be-

Vor diesem Hintergrund erscheint auch die Diskussion um den Status der Futura als temporale oder modale Formen in vielen Teilen überholt. Vgl. hierzu auch den Forschungsbericht bei Helland (1994: 212-222); vgl. ferner Martin (1981: 81f., 86); Fleischman (1982: 30f.); Palmer (1986: 209ff); Ludwig (1988: 99ff.). Für eine diachronische Betrachtung vgl. Bybee/Pagliuca/Perkins (1994: 95-97), die einen temporalen Ausgangswert postulieren, von dem dann die modalen Werte abgeleitet werden.

61 Damourette/Pichon (1936: 456ff.); Imbs (1960: 53f., 113); Wilmet (1976: 42-47); Klein (1980: 141ff.); Vet (1981a: 161); Fleischman (1982: 90); Togeby (1982: 388, 421f); Helland (1994: 226-233, bes. 231).

62 Als Ausdruck der Sprecherhypothese konkurriert das futur antérieur de probabilité mit der epistemischen devoirPeriphrase, die ja im Beispiel (3) "Ils ont dû avoir une expression horrifiée" ebenfalls verwendet wird. Zu den Differenzierungen im semantisch-pragmatischen Profil der beiden Formen vgl. Dendale (1994: 26ff.); Dendale/ Tasmowski (1994: 4-6); Schrott (1997: 304-310). 
schränkte kommunikative Garantie. Die durch das futur antérieur de probabilité so geleistete Versprachlichung einer Vermutung bezeugt auch die in beiden referierten Modellen postulierte Intensivierung der Sprecherzentrierung, da im Akt der Vermutung eine Sprecherillokution vollzogen wird. Die Futurität wird also nicht de re auf den Sachverhalt, sondern de dicto auf die Verifizierung und "prise en charge" durch den Sprecher bezogen und damit von der propositionalen Ebene des énoncé auf die textuell-expressive Ebene der énonciation verschoben. ${ }^{64}$

Für das Funktionieren des futur antérieur als futur de probabilité ist jedoch noch ein weiterer Mechanismus entscheidend, der auf der Anterior-Semantik der Form basiert. Auch beim modalen futur antérieur ist nämlich eine Relation zwischen abgeschlossenem Sachverhalt und Folgephase gegeben, wie anhand des nächsten Beispiels aufgezeigt werden soll, in dem über die Flucht eines jungen Mädchens Vermutungen angestellt werden:

(4) Jean, mon ami, vous vous êtes encore disputé avec votre fille, n'est-ce pas? Cet enfant aura pris la mouche et sera partie à Epoisses, à Bourbilly ou à Chastellux. Elle aura demandé l'hospitalité à ses cousins. [aristocrates, S.177]

Das Verschwinden des Mädchens, mit dem Sprecherin und Gesprächspartner im ego-hic-numc konfrontiert sind, wird durch die im futur antérieur de probabilité versprachlichten Hypothesen mit möglichen Handlungen verknüpft, die als Konsequenz das Verschwinden des Mädchens nach sich ziehen könnten.

Bei der modalen Verwendung wird also von einer in der origo gegebenen Folgephase auf einen vorzeitig zur Sprechsituation zu Ende geführten Sachverhalt geschlossen. Die bei dieser Vermutung hergestellte Relation zwischen Sachverhalt und Folgephase stellt nun eine Korrelation dar, die von der "present relevance"-Semantik des Anterior herrührt. Beim Verwendungstyp des futur antérieur de probabilité wird die "present relevance" als Relation zwischen Folgephase und vorzeitigem Sachverhalt umgedeutet zu einer Vermutung, bei der ein präsentischer Zustand auf eine wahrscheinliche Ursache zurückgeführt wird. Basis für diese Umdeutung ist die für den Anterior konstitutive Konfiguration von Sachverhalt und Folgephase, die als temporale Kontur unberührt bleibt, jedoch einer auf den Sprecher zentrierten Interpretation unterzogen wird: Eine Folgephase ist für einen Sprecher Indiz einer bestimmten, bereits abgeschlossenen Handlung, die als solche nicht mehr beobachtet werden kann, aber ihre Spuren hinterlassen hat. Der semantisch-pragmatische Wert der Vermutung kann in diesem Licht als Ergebnis metaphorischer und metonymischer Prozesse gesehen werden. Zum einen wird die Relation von Sachverhalt und Folgephase in einen anderen, distanten Kontext gerückt, zum anderen wird diese Relation durch die entsprechenden Kontexte des Vermutens metonymisch mit einer Semantik von Ursache und Wirkung pragmatisch angereichert.

Dieses Neuarrangement der semantischen Konfiguration beim futur antérieur kann durch einen besonderen Gebrauchstyp des futur simple untermauert werden, bei dem das futur simple stark restringiert in der Funktion futur de probabilité verwendet wird. Das futur simple ist im 
Französischen hier nur mit den Verben avoir und être belegt, ${ }^{65}$ also mit Verben, die exemplarische "states" ausdrücken: ${ }^{66}$

(5) Roger n'est pas venu: Il sera malade ou il aura des ennuis. ${ }^{67}$

Diese Beschränkung des futur simple de probabilité auf "states" ist als Reflex des futur antérieur zu erklären und als Versuch, die Anterior-Semantik mit anderen sprachlichen Mitteln nachzubilden. Die beim futur antérieur gegebene Relation zwischen abgeschlossenem Sachverhalt und Folgephase ist in der Basissemantik des futur simple nicht enthalten und wird durch die Auswahl entsprechender Situationstypen kompensiert - die Anterior-Semantik wird also gleichsam durch die Verbsemantik nachgeahmt und das futur simple erscheint in dieser Funktion als Reflex des futur antérieur.

Für das futur antérieur ergibt sich damit die Anterior-Semantik als unveränderte gemeinsame Basis der temporal-futurischen Verwendung und des Gebrauchs als futur antérieur de probabilité. So wie beim temporalen futur antérieur eine Folgephase aus einem abgeschlossenen Sachverhalt entsteht, so wird beim modalen futur antérieur de probabilité eine präsentische Folgephase vom Sprecher in der Sprechhandlung einer Hypothese mit einer (vermuteten) Ursache korreliert. Der Anterior und das modale futur antérieur de probabilité haben nun gemeinsam, daß ein Sachverhalt nicht per se dargestellt wird, sondern in Relation zu seinem Resultat. Da die Relation von Sachverhalt und Folgephase beim modalen Verwendungstyp nun als Inferenz eines Sprechers interpretiert wird, entspricht diese Funktion des futur antérieur der in der Forschung beschriebenen Kategorie des "inferential". ${ }^{68}$ Beim "inferential" wird die Korrelation von Sachverhalt und Folgephase fokussiert und mit nicht-temporalen Werten im Sinne einer Ursache-Wirkung-Relation angereichert. Diese Anreicherung basiert nun auf dem metonymischen Mechanismus der "context-induced reinterpretation". Denn eine temporal-aspektuelle "present relevance" wird kontextuell häufig mit kausalen Inferenzen verknüpft, die dann in die Bedeutung der Form integriert werden und den Funktionstyp des futur antérieur de probabilité konstituieren. Die Umdeutung hat aber auch eine metaphorische Komponente, denn temporaler Anterior und "inferential" sind auch durch eine analog-ikonische Struktur von Sachverhalt und Folgephase gekennzeichnet, für die gilt, daß der Sachverhalt selbst nicht beobachtbar ist und daher kognitiv der Hypothese verwandt ist. ${ }^{69}$ Diese Fokussierung der (kausalen) Inferenz stellt eine Interpretation des Sprechers dar und entspricht damit der Hypothese Traugotts, daß Bedeutungswandel mit einer Aufladung an Subjektivität und mit einer Zentrierung auf die Sprecherinstanz als deu-

Togeby (1982: 388)

Nach Cartagena (1981: 384ff.) findet sich eine ahnliche Restriktion auf Zustandsverben auch im Spanischen, wo das futuro de probabilidad vor allem in der 3 . Person mit Zustandsverben verwendet wird. Vgl. auch Bybee/Pagliuca/Perkins (1994: 51, 53, 202, 207)

Beispiel Kahn (1954: 84).

Comrie (1976: 108ff.); Traugott (1989: 48, 50); Anderson (1986: 275, 293); Aksu-Koç/Slobin (1986: 159ff.); Bybee/Pagliuca/Perkins (1994: 95-97).

Traugott (1989: 48, 50). Dieser Mechanismus der Umdeutung wird in der Forschung - so bei Comrie (1976: 110), Anderson (1986: 275, 293) und Aksu-Koc/Slobin (1986: 159ff.) - auch für andere Sprachen wie z.B. das Türkische, Georgische und Balkan-Slavische belegt. Weitere Beispiele fur den Mechanismus der Umdeutung bei Bybee/Pagliuca/Perkins (1994: 197-200). 
tende und ordnende Kraft innerhalb der Welt des Textes und der versprachlichten Sachverhalte einhergeht.

\subsection{Das futur antérieur der retrospektiven Beurteilung}

Der modale Funktionstyp des futur de probabilité stellt nun nicht den einzigen modalen Wert des futur antérieur dar. So finden sich im gesichteten Beispielmaterial häufig Verwendungen, in denen das futur antérieur vergangene Sachverhalte ausdrückt und diese Ereignisse rückblickend im Sinne einer Bilanz und Beurteilung darstellt. In den folgenden Beispielen soll aufgezeigt werden, wie beim modalen futur antérieur die Semantik von verschobener Verifizierbarkeit und Anterior-Relation eingesetzt werden kann, um eine Aussage als retrospektive Beurteilung zu markieren. Eine solche Nuance der Beurteilung tritt meist in kommentierenden Texten auf, die vergangene Ereignisse aus der Sicht des Sprechers schildern:

(6) Le dernier livre de Pierre Péan.

Les secrets du jeune Mitterrand.

Le journaliste enquêteur a minutieusement reconstitué la jeunesse du Président. [...].

Les historiens n'ont pas fini de fouiller les mystères de la vie de François Mitterrand. En tout cas, Pierre Péan les aura sacrément aidés. Même si, comme l'a écrit le Président, dans la revue Montalembert, alors qu'il était étudiant: "Le mystère de l'homme me paraît une colossale pièce montée." [NO 1556, S.32f.]

Die Enthüllungen Pierre Péans, als Buch frisch veröffentlicht, werden nicht als Faktum präsentiert, sondern in der subjektiv-beurteilenden Sicht des Sprechers wiedergegeben, der die Aufdeckungen als effektiv bewertet ("sacrément") und ihnen eine wichtige Rolle für die historische Aufarbeitung der Ära Mitterrand zuweist. Diese Beurteilung hat als subjektives Urteil des Sprechers nicht den Status eines Faktums und ist darin der verschobenen Verifizierbarkeit futurischer Sachverhalte verwandt.

Die von diesem Verwendungstyp des futur antérieur geleistete Beurteilung findet sich auch häufig bei der Schilderung historischer Ereignisse; das folgende Beispiel stammt aus der Rubrik "L'éditorial de Jean Daniel":

(7) Après le veto de la Grande-Bretagne, le désaveu d'une partie de son gouvernement et l'hostilité de toute la bourgeoisie française, Léon Blum cède. Mais devant l'angoisse populaire et parce qu'il est un démocrate jusqu'au bout des ongles, il comprend qu'il a des comptes à rendre. Il le fait dans son célèbre discours de Luna Park. Un discours qui est encore aujourd'hui l'objet de discussions, mais qui constitue l'un des moments les plus pathétiques d'un homme d'Etat. Rarement citoyens auront eu autant le sentiment de participer à une décision, à sa génèse et à ses conclusions, même lorsqu'ils désapprouvaient cette décision. [NO 1541, S.22]

Auch in diesem Beispiel, in dem der historische Kontext die retrospektive Markierung noch weiter verstärkt, liegt der Akzent nicht auf den historischen Ereignissen selbst, sondern auf einer subjektiven Sicht der berichteten Ereignisse. Das futur antérieur dient hier dazu, die beurteilenden Passagen von den Fakten der Rede Blums zu trennen. Léon Blums Rede wird nicht nur explizit als epochemachendes Demokratiebekenntnis charakterisiert, sondern gewinnt auch durch die Blickrichtung von der Gegenwart zurück in die Vergangenheit an Bedeutung. Die Retrospektive suggeriert, daß in diesem Zeitraum nur einmal so eine Rede gehalten wurde, woraus 
eine fokussierende Wirkung des futur antérieur folgt. Die bereits in den vorangehenden Beispielen besprochene rückschauende Perspektive der Beurteilung wird in diesem Kontext noch deutlicher: Aus der gegenwärtigen Situation heraus kann der Sprecher deutend historische Sachverhalte miteinander verbinden.

In der Mehrzahl der gesichteten Texte wie auch in den Beispielen (6) und (7) gilt das Urteil des Sprechers einem Dritten, doch kann die Beurteilung auch der eigenen Person gelten bzw. einer Gruppe, zu der der Sprecher sich zählt. Diese Spielart illustriert das folgende Beispiel, in dem eine Begebenheit aus dem Leben von Yves Montand berichtet wird:

(8) A Moscou, en juin 1990, Costa-Gavras et moi avons assisté auprès de Montand à la première projection publique de "l'Aveu", qu'avaient organisée "les Nouvelles de Moscou". Lorsque les lumières se rallumèrent, un silence lourd, foisonnant de douleurs intimes, de cris retenus, de larmes refoulées, régnait sur l'assistance. Ensuite des spectateurs de tous âges se levèrent. Ils s'adressaient à Montand, bien sûr. C'est lui qu'ils remerciaient pour ce film qui les déchirait, tout en les réconciliant avec leur mémoire authentique, si longtemps censurée. A la fin, Montand m'a regardé: voilà, semblait-il me dire, nous aurons quand même vécu cela. Et nous l'aurons vécu ensemble. Loin des polémiques, et parfois des médisances parisiennes et partisanes, l'accueil du public de Moscou était pour Montand la meilleure justification de son itinéraire politique. [NO 1541, S.7]

Der Text beschreibt die öffentliche Vorführung eines Films, der mit dem kommunistischen System hart ins Gericht geht und nun nach vielen Jahren offiziell in Moskau gezeigt wird. Montands Äußerung ist in diesem Kontext keine Feststellung, sondern eine Beurteilung der ungewöhnlichen Hommage, auf die er schon nicht mehr hoffte. Wie schon in (7), so ist auch hier die Nuance der Retrospektive dominant, die Beurteilung Montands hat den Charakter einer Bilanz, der verlorengeht, wenn man das futur antérieur durch ein ebenfalls mögliches passé composé ersetzt. Dieser Kommutationstest, der bei analogen Beispielen zum gleichen Ergebnis führte, ist nun ein Indiz dafür, daß die Nuance der retrospektiven Beurteilung nicht allein aus den Kontexten resultiert, sondern daß das semantisch-pragmatische Potential des futur antérieur hier maßgeblich beteiligt ist.

Der beschriebene Verwendungstyp der retrospektiven Beurteilung erklärt sich durch mehrere ineinander verflochtene Mechanismen. Analog zum futur antérieur de probabilité wird die Futurität metaphorisch zur Nicht-Verifizierbarkeit umgedeutet, die dann die Faktizität der vergangenen Sachverhalte mindert und den Boden für den pragmatischen Wert einer Beurteilung bereitet. Der metaphorische Prozeß wird auch bei diesem Verwendungstyp durch einen metonymischen Mechanismus ergänzt. Wie schon beim futur antérieur de probabilité so wird auch hier die metaphorische Umdeutung der Futurität zur Nicht-Verifizierbarkeit durch die Kontiguität von Kontexten der Vergangenheitsreferenz ausgelöst, so daß auch hier Metapher und Metonymie Hand in Hand gehen. Analog zum futur antérieur de probabilité verliert die Anterior-Form auch bei diesem Typ die propositionale Funktion der futurischen Situierung und gewinnt einen textuell-expressiven Wert. Wie der Ausdruck einer Sprecherhypothese, so stellt auch die Beurteilung einen Transfer von der propositionalen auf die textuell-expressive Funktionsebene dar. Die im futur antérieur versprachlichten Sachverhalte werden im Sinne einer Diskursmarkierung als Beurteilungen von den berichteten Fakten abgesetzt. Urheber der Beurteilung ist ebenfalls der Sprecher, der damit auch bei dieser modalen Verwendung als zentrale Instanz fungiert, in deren "univers de croyance" die referierten Sachverhalte gespiegelt und dann gleichsam in subjektiver Brechung auf den Text zurückgeworfen werden. 
Diese Umdeutung der Futurität zum Ausdruck einer Vermutung oder Beurteilung kann daher auch durch eine Differenzierung verschiedener Ebenen der Modalität, der "modalité d'énoncé" und der "modalité d'énonciation" noch genauer gefaßt werden. ${ }^{70}$ So versprachlicht die "modalité d'énoncé" die epistemische Gewißheit und den Wahrheitsgehalt, den die Gesprächspartner einem Sachverhalt zuordnen, während die "modalité d'énonciation" auf Illokutionen und Relationen der Gesprächspartner untereinander bezogen ist. " Der beschriebene Mechanismus der metaphorischen Umdeutung erscheint so als Verschiebung von der Ebene des énoncé auf die Ebene der énonciation. Während bei temporal-futurischen Verwendungen die Futursemantik auf die Sachverhalte des énoncé referiert, bezieht sich die Semantik der Futura bei modalen Verwendungstypen auf die Ebene der énonciation und die auf dieser Ebene vollzogenen Illokutionen und Interaktionen.

Neben der Futurität und epistemischen Modalität des futur antérieur spielt noch ein zweiter Merkmalkomplex eine wichtige Rolle. Entscheidenden Anteil am Verwendungstyp der retrospektiven Beurteilung hat, wie schon beim futur de probabilité, die für das futur antérieur konstitutive Relation zwischen abgeschlossenem Sachverhalt und Folgephase. In den zitierten Beispielen erfolgen die Beurteilungen der in der Vergangenheit abgeschlossenen Sachverhalte aus der Situation des Sprechers im ego-hic-nunc heraus. Wie beim Ausdruck einer Hypothese, so werden auch bei Beurteilungen Verbindungslinien zwischen abgeschlossenen Sachverhalten und der Gegenwart hergestellt. Die Gewichtung und Bewertung in der Retrospektive erfolgt aus der Situation des ego-hic-munc heraus. Die beim Anterior gegebene Relation zwischen Sachverhalt und Folgephase manifestiert sich also in der Retrospektivität der Beurteilung als vom Sprecher etablierte Relation zwischen gegenwärtigen Gegebenheiten und einem in der Vergangenheit zu Ende geführten Sachverhalt. Diese Umdeutung der Anterior-Semantik wird durch die beim futur antérieur gegebene Fokussierung der Folgephase begünstigt. Die Hervorhebung impliziert nämlich eine 'Blickrichtung' von der Folgephase auf den Sachverhalt und enthält damit in nuce die Semantik der Retrospektive, die dann für das modale futur antérieur entscheidend wird. Eine solche retrospektive Betrachtung ist dann häufig mit bilanzierend-beurteilenden Betrachtungsweisen verknüptt, die als konversationelle Implikaturen dann durch den beschriebenen metonymischen Mechanismus in die Semantik der Form integriert werden können. Dieser der Retrospektive inhärente Charakter der Beurteilung entspricht in seinem Potential nun der metaphorisch gewonnenen Semantik der Nicht-Verifizierbarkeit, so daß beide Mechanismen sich im Sinne einer positiven Rückkopplung verstärken könnten. Wie schon beim futur de probabilité, so bleibt auch beim futur antérieur der Retrospektive die semantische Konfiguration von Sachverhalt und Folgephase an sich erhalten, wird jedoch unter dem Einfluß der Sprecherinstanz neu interpretiert.

Das futur antérieur in seiner Wirkung der retrospektiven Beurteilung wird vor allem kontrastiv zu anderen Tempora deutlich. Das folgende Beispiel schildert, wie im Sommer 1914 die angeordnete Mobilmachung im Kreis der Sozialisten diskutiert wird. Einen alten Mann regt die Krise an, eine persönliche Bilanz zu ziehen:

70 Jacques (1983: 66f.)

71 Verwandte Differenzienungen der Modalităten finden sich bei: Martin (1983: 129ff.); Parret (1983: 83ff., 90ff.); Nolke (1985: 104f., 113) und (1989: 47-51); Siewierska (1991: 115ff., 123ff.). 
(9) "J'aurai eu trois sombres dates dans mon existence", commença-t-il, sur ce ton appliqué qu'il prenait en publique, à ses cours, (et qui faisait rire aux étudiants: "Phi-Phi s'écoute parler"). "La première a révolutionné mon adolescence; la seconde a bouleversé mon âge mûr; la troisième empoisonnera sans doute ma vieillesse ..."

Antoine le dévisageait comme pour l'inciter à poursuivre:

"La première, c'est quand l'enfant provincial et pieux que j'étais, a découvert, une nuit, en lisant à la file les quatre Evangiles, que c'était un tissu de contradictions ... La seconde, c'est quand je me suis convaincu qu'un vilain Monsieur, qui s'appelait Esterhazy, avait fait une saloperie, qui s'appelait 'le bordereau', et que, au lieu de le condamner, on s'acharnait à torturer à sa place un monsieur qui n'avait rien fait, mais qui était juif ..."

- "Et la troisième", l'interrompit Antoine, avec un triste sourire, "c'est aujourd'hui ..."

- "Non ... la troisième, c'est il y a huit jours, quand les journaux ont donné le texte de l'ultimatum, quand j'ai vu se dessiner la partie de billard ... Quand j'ai compris que c'étaient les peuples qui allaient faire les frais du carambolage ..." [Thibault IV, S.278]

Das futur antérieur leitet hier eine Bilanz ein, die im folgenden durch Vergangenheitstempora fortgesetzt wird. Der Sprecher schildert drei einschneidende Erlebnisse, die zunächst nicht an sich als Fakten erzählt, sondern nur im Hinblick auf ihre Wirkung auf den Sprecher in den verschiedenen Altersstufen präsentiert werden. Erst nach Darlegung dieses subjektiven Erlebens werden die persönlichen und politischen Ereignisse selbst geschildert. Für diese Herangehensweise des Erzählers gibt das modale futur antérieur nun gleichsam die Tonart an und signalisiert, daß der Sprecher gelebte Geschichte aus seinem persönlichen Blickwinkel präsentieren wird. Ersetzt man das futur antérieur durch ein passé composé, dann geht dieses Signal verloren, ebenso wie die Differenzierung zwischen den historischen Fakten und der Wirkung, die der Sprecher diesen Fakten rückblickend beimißt.

In den bisher behandelten Textausschnitten manifestiert sich die Nuance der Beurteilung in Kontexten mit bewertend-kommentierendem Charakter. Kommutationstests und Sprecherbefragungen weisen jedoch nach, daß das Potential der retrospektiven Beurteilung von der AnteriorForm herrührt und durch die Kontexte lediglich verstärkt wird. Das modale futur antérieur tritt nun auch in Textpassagen auf, die keinen kommentierenden Charakter aufweisen und in denen ein modales futur antérieur daher nicht erwartbar scheint:

(10) Transfert des cendres d'André Malraux au Panthéon le 23 novembre 1996.

André Malraux au Panthéon.

Il n'aura pas reçu le Prix Nobel, dont Albert Camus le déclarait plus digne que lui-même. Il n'aura pas siégé à l'Académie française, où le Général de Gaulle, à la libération, eût souhaité qu'il entrât - avec Gide, Bernanos, Aragon ... Mais le voilà au Panthéon! [Revue de l'Amopa 135, 1996, S. 8]

In diesem Text versprachlicht das futur antérieur Fakten aus der Biographie Malraux', die nur geringfuigig beurteilenden Charakter haben, so daß die Präsenz des Sprechers als beurteilende Instanz auf den ersten Blick schwach ausgeprägt zu sein scheint. Eine Kommutation durch das passé composé und eine Sprecherbefragung ergeben jedoch, daß auch in diesem berichtenden Text der modalisierte Anterior eine bewertende Rückschau etabliert und das Specher-Ich konstituiert. Im Unterschied zum passé composé vermag das futur antérieur des Originals so nämlich zu suggerieren, daß Malraux für die (zu Unrecht) nicht erfahrenen Ehrungen nun durch die Aufnahme in das Panthéon späte Gerechtigkeit widerfährt. 
Einen analog gelagerten Fall liefert der folgende Textausschnitt, in dem das Massaker von Hebron und die von ihm ausgelösten Reaktionen behandelt werden:

(11) Ils vont désarmer des colons extrémistes. Ils vont libérer (pas assez) des prisonniers palestiniens. Ils acceptent des observateurs civils de l'ONU. C'est mieux. C'est bien. Mais ...

... Mais il nous aura fallu entendre des hommes, des religieux, des juifs - oui, des juifs - affirmer que le tueur d'Hébron était un "héros". Nous aurons entendu cela. Nous aurons entendu déclarer que massacrer une foule désarmée dhommes en prière constituait un "acte pieux". Ceux-là sont peut-être plus monstrueux que le tueur lui-même. [NO 1530, S.24]

Die im futur antérieur versprachlichten Aussagen geben die Äußerungen extremistischer Siedler wieder und scheinen daher kaum als Beurteilungen des zitierenden Sprechers interpretierbar zu sein. Die Tempusverwendung erklärt sich aber, wenn man berücksichtigt, daß der Textabschnitt aus der Rubrik "L'éditorial de Jean Daniel" stammt, in der Daniel aus subjektiver Sicht die Nachrichten der Woche kommentiert. Dieser beurteilende Charakter des Textes findet seinen Ausdruck im modalen futur antérieur, das durch seine abgeschwächte Assertion die Subjektivität des Kommentars signalisiert. Diese Deutung wird auch entscheidend durch die Tatsache gestützt, daß die ebenfalls im Dossier zum Massaker von Hebron enthaltenen berichtenden Texte, die eine Rekapitulation der Ereignisse ohne Wertung liefern sollen, kein modales futur antérieur enthalten. Die Verwendung des futur antérieur ist hier also nicht durch wertende Elemente des Kontextes bestimmt, sondem wird durch den wertenden Charakter der Textsorte insgesamt ausgelöst. So wie das modale futur antérieur einzelne Textpassagen als Beurteilungen und subjektive Urteile markieren kann, so kann auch eine kommentierende Textsorte insgesamt durch diese futur antérieur-Verwendung noch deutlicher als Beurteilung markiert werden.

Im nächsten Abschnitt sollen die beschriebenen Verwendungstypen des futur antérieur und die metonymischen und metaphorischen Prozesse, die diese Funktionen verknüpfen, im Sinne der eingangs erläuterten synchronischen Kategoriendynamik diskutiert werden.

\section{Zur Dynamik des futur antérieur}

Die vorangegangene Sichtung der Verwendungstypen des futur antérieur zeigt, daß einer temporal-futurischen Verwendung der Anterior-Form zwei modale Verwendungstypen gegenüberstehen, die als Ausprägungen eines modalisierenden Mechanismus aufgefaßt werden können. Die ausgehend von Beispielanalysen erarbeiteten Funktionsprofile des futur antérieur de probabilité und des futur antérieur der retrospektiven Beurteilung weisen deutliche Gemeinsamkeiten auf. Beide Verwendungstypen basieren einmal auf der Semantik der Nicht-Verifizierbarkeit, die gleichsam die modal-epistemische Kehrseite der Futurität darstellt und die in Kontiguität zu Kontexten der Vergangenheitsreferenz beide Male eine metaphorische Umdeutung erfährt. Die beschriebenen modalen Verwendungen nutzen in gleicher Weise die aspektuelle Markierung des Anterior durch eine Umdeutung der Relation von abgeschlossenem Sachverhalt und Folgephase, die als semantische Konfiguration aber intakt bleibt. Da die Struktur von Sachverhalt und Folgephase erhalten bleibt und allein durch eine veränderte Fokussierung und semantische 'Grenzziehung' verändert wird, weist dieser Typ von Bedeutungswandel eine Analogie zur Reanalyse als 
Erklärungsmodell auf. Bei der Reanalyse werden ja aufgrund eines gegebenen Inferenzpotentials syntagmatische Relationen von Elementen eines Ausdrucks ohne ausdrucksseitige Veränderungen modifiziert. Eine auf abstraktem Niveau ähnliche inferenzbedingte Umdeutung findet nun auch in der Semantik des futur antérieur statt. Die Relation von abgeschlossenem Sachverhalt und daraus resultierender Folgephase beinhaltet als Inferenzpotential dieser Struktur die semantisch-pragmatischen Werte der Vermutung und der Beurteilung aus der Retrospektive. Da die Bausteine der semantischen Konfiguration erhalten bleiben und lediglich in ihrem Bezug zueinander umgedeutet und neu arrangiert werden, könnte man in diesem Typ des Bedeutungswandels bzw. der Polysemie eine 'semantische Reanalyse' sehen - die semantische Konfiguration bleibt in ihrer Struktur unberührt, wird aber umgedeutet und erfült dann auch neue Funktionen.

Ordnet man die temporalen und modalen Verwendungstypen vor der Folie des Modells propositionaler und textuell-expressiver Funktionen ein, dann erscheint das temporal-futurische futur antérieur als propositionale Verwendung, die Sachverhalte situiert, während das futur antérieur de probabilité ebenso wie das futur antérieur der retrospektiven Beurteilung als Ausdruck der Sprecherhaltung textuell-expressive Funktionen haben. In beiden modalen Typen konstituiert sich die Sprecherinstanz mit ihrem "univers de croyance" in der Textwelt als beurteilende und ordnende Instanz, so daß beide Verwendungstypen durch den gemeinsamen Nenner der Beurteilung verbunden sind. Auch das futur de probabilité kann nämlich als Form der Beurteilung gedeutet werden, denn der Ausdruck einer Vermutung stellt auch immer eine Bewertung nach dem Kriterium der Wahrscheinlichkeit dar. ${ }^{72}$

Die Darlegung der Funktionstypen belegt ferner, daß das semantisch-pragmatische Potential des futur antérieur das Ergebnis metaphorischer und metonymischer Prozesse ist, die eng ineinander verzahnt sind. So wird das temporale Konzept der Futurität umgedeutet zu verschobener Verifizierbarkeit und einer Sprecherdistanz, die sich dann in den textuell-expressiven Funktionen der Hypothese und der Beurteilung manifestieren. Ausgelöst wird diese Metapher durch die Verwendung der Form in nicht-futurischen Kontexten, so daß in dieser Kontiguität ein metonymischer Faktor zum Tragen kommt. Ein metonymischer Mechanismus modifiziert auch die Anterior-Semantik, die als temporale Relation von Folgephase und vorangegangenem Sachverhalt metonymisch durch pragmatische Inferenzen angereichert wird. Die so belegte Engfuhrung metaphorischer und metonymischer Prozesse, die sich in allen untersuchten Bereichen durchdringen, spricht fuir Modelle der Grammatikalisierung und des Bedeutungswandels, die weniger die 'Aufgabenteilung' dieser Prozesse als vielmehr ihre Verflochtenheit wiedergeben

Die textuell-expressiven Funktionen des modalen futur antérieur bauen damit auf dem Grundwert der verschobenen Verifizierbarkeit auf und belegen so, daß pragmatisch-textuelle Werte der Futura von der temporal-aspektuellen Basissemantik der Formen ableitbar sind und durch Interaktion mit dem Kontext entstehen. Die Untersuchung untermauert so die Hypothe-

72 In der Forschung wird das futur antérieur der Beurteilung meist isoliert betrachtet und nicht auf Beruhrungspunkte zum futur antérieur de probabilité hin untersucht. Meiner Deutung verwandte Charakterisierungen des futur antérieur als künftiges Urteil des Sprechers bzw. als Urteil kunftiger Generationen finden sich bei Rauhut (1957: 424-428), Heger (1963: 113f), Wilmet (1976: 48-51); Togeby (1982: 421). Ferner wird der Ausdruck vergangener Sachverhalte durch das futur antérieur hinsichtlich der aspektuellen Markierung auch als kompensatorische Ergănzung der Vergangenheitstempora gedeutet; vgl. Imbs (1960: 111f); Wilmet (1976: 167ff., 173); Vet (1980: 88f.); Steinmeyer (1987: 119ff., 124f.); Gobert/Maisier (1995: 1008). 
se, ${ }^{73}$ daß sich diskursiv-pragmatische Werte aus der temporal-deiktischen Tempusfunktion herleiten, und widerspricht den Ansätzen von Weinrich (1982) ${ }^{74}$ und Hopper (1979a, 1979b, 1982a), ${ }^{75}$ die die Diskursfunktionen als primäre Größen ansetzen, von denen sich dann die temporal-deiktischen Funktionen ableiten sollen. Die Kopräsenz der Verwendungstypen bestätigt die Ausgangshypothese, daß propositionale, textuelle und expressive Funktionen nicht als zeitlich aufeinanderfolgende Entwicklungsstadien aufzufassen sind, sondern als Funktionstypen, die sich aus der Basissemantik der Anterior-Form futur antérieur ableiten lassen und die durch metaphorische und metonymische Prozesse eng miteinander verknüpft sind. ${ }^{76}$

Eine synchronisch angelegte Untersuchung kann das Netzwerk temporal-aspektueller und modalisierter Funktionen durch Beispielanalysen erfassen und so die semantisch-pragmatischen Profile der Verwendungstypen erstellen. Um die konstatierte Kopräsenz von Funktionen als eine Entwicklungsstufe der Tempusform besser beurteilen zu können, soll die Synchronie durch einen auf die Forschung gestützten Ausgriff in die Diachronie ergänzt werden. Seit Diez (1882) und Tobler $(1886)^{77}$ finden sich in der Forschung Hinweise auf eine Verwendung des futur antérieur mit Vergangenheitsreferenz, die dem hier beschriebenen futur antérieur der retrospektiven Beurteilung entspricht. Dieser Verwendungstyp ist bereits im Altfranzösischen belegt und hat sich bis ins heutige Französisch erhalten. ${ }^{78}$ Der semantisch-pragmatische Wert einer Beurteilung in der Retrospektive wird besonders von Tobler (1886) ${ }^{79}$ anhand von altfranzösischen Beispielen dokumentiert, und auch die fokussierende textuelle Wirkung des retrospektiven futur antérieur wird bei der Analyse altfranzösischer Textbeispiele herausgearbeitet. ${ }^{80}$ In der späteren Forschung wird dann die retrospektive Beurteilung meist verengend als ein vorweggenommenes Urteil der Nachwelt beschrieben - eine Erklärung, die dem retrospektiven Blickwinkel gerecht wird, nicht jedoch der Besonderheit, daß das Urteil ein Sprecherurteil aus der Perspektive des ego-hic-nunc ist. ${ }^{81}$ Diese Modifizierung der Beurteilung als Urteil der Nachwelt scheint mir auch durch das in diesen Beiträgen diskutierte Beispielmaterial bedingt zu sein. So legen etwa analysierte Beispiele aus dem Rolandslied die Perspektive der Nachwelt nahe, wenn abgeschlossene (Helden)-

73

4 Weinrich (1982: 160-162). Die Tempora werden als Ausdruck der Tempus-Register Erzählen ("entspannte Gelassenheit") und Besprechen ("gespannte Hinwendung") beschrieben, die Sprecherhaltung erscheint als Teil der Tempussemantik (1982: 160-162).

75 Markierungen fur Vordergrund und Hintergrund in Texten. Vet (1991: 7f., 12-23) versucht, die Dichotomie von
"foreground" und "background" in feineren Abstufungen zu beschreiben. 210f.); Gamillscheg (1957: 389f.); Rauhut (1957: 222ff., 225-228); vgl auch den Forschungsbericht zur alteren Forschung bei Wilmet (1976: 41 f.). In den romanischen Sprachen ist diese Verwendung nach Tobler (1886: 207 209) und Lerch (1928: 103f.) nur im Franzósischen und im Altprovenzalischen belegt.

So konstatiert Diez (1882: 969f.) eine markierte Raschheit der Handlung, Tobler (1886: 209) die Markierung einer hohen Quantität oder Qualităt durch ein häufiges mout und tant. Vgl. auch Rauhut (1957: 225f.); Wartburg/ Zumthor (1947: 210f.).

81 Lerch (1928: 103f.); Gamillscheg (1957: 389f); Wartburg/Zumthor (1947: 210f.); Rauhut (1957: 225-228). Rauhut bezieht die Sprecherinstanz starker ein, wenn er feststellt, daß das Urteil von der Nachwelt oder vom Sprecher gefallt werden kann. 
Taten von einem Sprecher beurteilt werden, der gleichsam das Sprachrohr künftiger Generationen ist. ${ }^{82}$ Eine mögliche Erklärung für den mehrfach konstatierten Wert der Beurteilung durch die Nachwelt wäre auch, daß diese Bedeutung am Anfang der Entwicklung dieser modalen Funktion des futur antérieur steht. Eine in diesem Zusammenhang plausible, noch zu verifizierende Hypothese wäre etwa, daß der Wert der retrospektiven Beurteilung zunächst gebunden an Kontexte auftrat, die eine historische Dimension der Beurteilung durch künftige Generationen implizierten, und dann allmählich eine Bedeutungserweiterung erfuhr und sich zunehmend von dieser kontextuellen Anbindung löste. Eine solche Hypothese kann allerdings nur dann das Stadium der "conjectural history" verlassen, wenn sie durch Corpusauswertungen verschiedener Sprachstadien gestützt wird. Eine solche diachronische Ausweitung nach dem Muster der auf die Synchronie beschränkten Textanalysen ist auch unumgänglich, wenn Schwerpunktverlagerungen zwischen temporalen und modalen Funktionen des futur antérieur zu diskutieren sind, also etwa die Annahme, daß das futur antérieur sich von einer temporal-futurischen zu einer modalen Form entwickelt.

Die bereits im Altfranzösischen konstatierte Verwendung des retrospektiven futur antérieur belegt ferner, daß es sich bei diesem modalen Gebrauchstyp nicht um eine neuere Entwicklung handelt, sondern um eine Erscheinung, die im System des Französischen über eine längere Periode der Sprachentwicklung hin ihren Platz hat. Das Französische verfügt damit über einen Funktionstyp, der es dem Sprecher erlaubt, sich als beurteilende Instanz zu manifestieren, und so dem Streben des Sprechers nach Expressivität entgegenkommt. Diesen expressiven Wert dokumentiert auch das ausgewertete Corpus. Geht man von den Konzepten der Nähesprache und der Distanzsprache mit den sie konstituierenden Kommunikationsbedingungen aus, ${ }^{83}$ dann fält auf, daß im Französischen der Gegenwart das futur antérieur der retrospektiven Beurteilung sich vorwiegend in Textsorten zu manifestieren scheint, die im Sinne von Koch/Oesterreicher $(1985,1990)$ distanzsprachlich markiert sind. ${ }^{84}$ In diesen distanzsprachlichen Kontexten sind Innovationen unter anderem durch die Selbstdarstellung des Sprechers im Rahmen der Alttagsrhetorik motiviert. Dieser Motor könnte auch das futur antérieur der retrospektiven Beurteilung in seiner Position stärken bzw. vorantreiben, da die modale Funktion durch die Einbringung der urteilenden und rückschauenden Sprecherinstanz ja ganz im Sinne der Alltagsrhetorik wirkt. ${ }^{85}$ Diese mögliche Funktionsausweitung des retrospektiven futur antérieur deutet sich auch in den Beispielanalysen an. So hat dieser modale Typ in zahlreichen Beispielen zwar starke Affinität zu kommentierenden Kontexten, das futur antérieur kann diese Funktion jedoch auch erfullen, wenn die wertende Sprecherperspektive auf dem sehr abstrakten Niveau einer Textsorte angesiedelt ist und sich 'lokal' im Text nur schwach manifestiert. Damit stellt das futur antérieur der retrospektiven Beurteilung eine textuelle Funktion dar, die auch ohne Stützung durch beurteilende Kontexte funktioniert.

82

83 lichkeit wird durch folgende graduelle Parameter bestimmt: Öfentlichkeit, Vertrautheit der Partner, emotionale Beteiligung, der Grad der Situations- und Handlungseinbindung von Kommunikationsakten, die physische Nahe oder Distanz der Gesprächspartner, Kooperation, Diaiogizităt, Spontaneität, Themenfixierung und Referenzbezug auf die origo.

84 Koch/Oesterreicher (1985: 19-24) und (1990: 8-12).

85 
Vor diesem Hintergrund erscheint es gerechtfertigt, die Hypothese aufzustellen, daß das futur antérieur der retrospektiven Beurteilung einen sich allmählich ausbreitenden Typ darstellt, so daß sich innerhalb der Verwendungstypen der grammatikalisierten Kategorie futur antérieur eine Verlagerung von temporalen zu modalen Verwendungen abzuzeichnen scheint. Diese Hypothese kann jedoch im Rahmen einer synchronisch angelegten Untersuchung nur mit beschränkter kommunikativer Regreßpflicht abgegeben werden, da ein einziger synchroner Querschnitt nicht ausreichend für eine Prognose erscheint. Erst in einem weiteren Schritt der Ausweitung in die Diachronie kann das synchronisch erarbeitete Netzwerk auch als zeitliche Entwicklung entfaltet werden, die dann Funktionsverschiebungen des futur antérieur aufzeigt.

Für eine solche diachronische Untersuchung leistet das synchronisch umrissene pragmatische Potential des futur antérieur eine wichtige Hilfestellung, wenn pragmatische Verwendungstypen früherer Entwicklungsstadien ausgelotet werden und dabei auch die pragmatische Motivation für den Sprachwandel beleuchtet werden soll. ${ }^{86}$ Die in der Grammatikalisierungsforschung gestellte Frage nach der pragmatischen Motiviertheit von Sprachwandel bewegte auch gerade im Falle des futur antérieur die schon zitierte ältere Forschung. ${ }^{87}$ In der Tradition der idealistischen Philologie deutet Lerch (1928) ${ }^{88}$ das retrospektive futur antérieur als Manifestation der französischen Mentalität, die das besondere Streben der Franzosen nach Ruhm und Anerkennung abbildet: Mit dem futur antérieur der Beurteilung haben sich die Franzosen eine Form geschaffen, die es ihnen erlaubt, schon in der Gegenwart künftigen Ruhm zu evozieren. Auch Lerch (1928) sucht also nach den Sprecherstrategien, die Grammatikalisierungsprozesse motivieren und vorantreiben, seine Erklärung erscheint allerdings aus Sicht der heutigen Linguistik als falsche Antwort auf eine richtig gestellte Frage. Diese Frage nach der Motiviertheit sprachlicher Formen stellt sich nun aber nicht nur Linguisten, sondern beschäftigt auch Sprecher, die über das Sprechen und Handeln anderer Sprecher nachdenken.

Diese Beschäftigung mit Sprache dokumentiert der folgende Ausschnitt aus der Glosse "Vergangene Zukunft". ${ }^{99}$ Der Verfasser kommentiert hier das Festhalten der Franzosen am überholten System des "Minitel", das als "vergangene Zukunft" eine Modernisierung der Informationstechnologien in Frankreich verhindert:

(12) Aus der Wellblechauto- und Gauloise-Ära der Pompidou-Jahre war das Land unter Giscard im Eiltempo technologisch auf Vordermann gebracht worden; unter Mitterrand begann es schon, seine Geschichte in der Vorzukunft zu konjugieren. Alle Welt blickte gebannt auf die franzossischen Tausendsassas, die auf ihren Minitels klimperten und Telefonnummern, Zugverbindungen oder ihren Bankkontostand auf den Bildschirm zauberten. Doch hat die Geschichte die Unsitte, daß sie weitergeht. [...] So hat Frankreich auf dem flimmernden Gelände der elektronischen Medien sich bereits sein Monument gebaut, das nun zu wanken beginnt. Für das Land mit dem Talent zur vorauseilenden Erinnerung war das Neue freilich von jeher auch dann schon Erinnerungsstuck, als es noch Gebrauchsobjekt war. [FAZ 30.08.1997, S.31]

Der in französischer Grammatik offensichtlich beschlagene Autor verknüpft hier ganz in Lerchscher Manier das Talent der Franzosen zur "vorauseilenden Erinnerung" mit der entsprechenden,

86 Zu dieser Fragestellung vgl. auch den Beitrag von Ulrich Detges in diesem Band.

87 Das Interesse der Forschung weckte dabei auch die Tatsache, daß das futur antérieur der Beurteilung nach Tobler (1886: 207-209) und Lerch (1928: 103f.) nur im Altfranzósischen und im Altprovenzalischen belegt ist.

88 Lerch (1928: 103).

89 Den Hinweis auf diese Glosse verdanke ich Franz Lebsanft. 
hier "Vorzukunft" genannten Tempusform. Das Textbeispiel belegt damit zum einen die Relevanz der Fragestellung nach der Motivation sprachlicher Formen auch außerhalb der Linguistik und illustriert zugleich, daß die Erklärungsmuster der Vossler-Schule bei reflektierenden Sprechern lebendig sind.

Kehrt man nach diesen tastenden Versuchen, Tendenzen eines sich entfaltenden Bedeutungswandels aufzuspüren, wieder zu der zugrundegelegten synchronischen Sichtweise zurück, dann erscheinen die untersuchten temporalen und modalen Funktionen als eng vernetzte Verwendungstypen. Temporale und modale Verwendungen schöpfen aus der gleichen Quelle, nämlich der Basissemantik des Anterior, die dann auf propositionaler und textuell-expressiver Ebene durch unterschiedlich starke Zentrierung auf die Sprecherinstanz differenziert genutzt wird. Das etablierte Netzwerk temporaler und modaler Bedeutungen läßt sich also auf eine semantische Konfiguration zurückführen, die durch die eng verflochtenen Mechanismen von Metapher und Metonymie modifiziert wird. Die metaphorischen Übergänge werden in dieser Perspektive dann nicht als zeitliche Abfolge gesehen, sondern als kopräsente Verwendungstypen. Die in der Semantik des futur antérieur gegebenen Komponenten - die Relation von Folgephase und abgeschlossenem Sachverhalt, die retrospektive Blickrichtung und die Duplizität von Futurität und Nicht-Verifizierbarkeit - bilden Bausteine, aus deren Konfigurationen die einzelnen kopräsenten Verwendungstypen hergeleitet werden können. So bilden die semantischen Bausteine der grammatischen Kategorie keine statische Struktur, sondern beinflussen sich wechselseitig und werden dann in ihrer Gesamtheit noch durch die jeweiligen Verwendungskontexte beeinflußt. Die Untersuchung des futur antérieur bestätigt so nicht nur das eingangs beschriebene Konzept der Kategorie als im Fluß befindlicher Einheit, sondern vermag auch aufzuzeigen, durch welche 'Fließrichtungen' die einzelnen semantisch-pragmatischen Komponenten charakterisiert sind.

\section{Literatur}

\section{Quellen}

Thibault IV = Martin du Gard, Roger: Les Thibault. Vol. IV. L'été 1914 (suite). - Paris 1936, Nachdruck Paris 1972 Editions Gallimard.

FAZ $=$ Frankfurter Allgemeine Zeitung vom 30.08.1997.

NO $=$ Le Nouvel Observateur. Exemplare aus den Jahrgängen 1990-1996.

Revue de l'Amopa 135 vom Dezember 1996.

aristocrates $=$ Saint Pierre, Michel de: Les aristocrates - Paris 1954: Editions de la Table Ronde .

\section{Forschung}

Abraham, Werner (1992/93): "Einleitung zum Thema dieses Bandes. Grammatikalisierung und Reanalyse: Einander ausschließende oder ergänzende Begriffe?" - In: Folia Linguistica Historica 13, 7-26.

- / Janssen, Theo (Hgg.) (1989): Tempus - Aspekt - Modus. Die lexikalischen und grammatischen Formen in den germanischen Sprachen. - Tübingen: Niemeyer (=Linguistische Arbeiten 237). 
Aksu-Koc, Ayhan A. / Slobin, Dan I. (1986): "A Psychological Account of the Development and Use of Evidentials in Turkish". - In: W. Chafe, J. Nichols (eds.): The Linguistic Coding of Epistemology (Norwood: Ablex Publishing Corporation) (= Advance in Discourse Processes 20) 159-167.

Anderson, Lloyd B. (1986): "Evidentials, Paths of Change and Mental Maps. Typologically Regular Asymmetries". - In: W. Chafe, J. Nichols (eds.): The Linguistic Coding of Epistemology (Norwood: Ablex Publishing Corporation) (= Advance in Discourse Processes 20) 273-312.

Anttila, Raimo (1972): An Introduction to Historical and Comparative Linguistics. - New York: Macmillan

Bybee, Joan L. / Dahl, Östen (1989): "The Creation of Tense and Aspect Systems in the Languages of the World". In: Studies in Language 13, 51-103.

- / Pagliuca, William (1985): "Cross-linguistic Comparison and the Development of Grammatical Meaning". - In: J. Fisiak (ed.): Historical Semantics - Historical Word-Formation (Berlin, New York: Mouton) (= Trends in Linguistics: Studies and Monographs 29) 59-83.

- / Pagliuca, William / Perkins, Revere D. (1991): "Back to the Future". - In: E. C. Traugott, B. Heine (eds.): Approaches to Grammaticalization. Vol. II (Amsterdam, Philadelphia: John Benjamins) (= Typological Studies in Language 19) 17-58.

- / Pagliuca, William / Perkins, Revere D. (1994): The Evolution of Grammar. Tense, Aspect and Modality in the Languages of the World. - Chicago, London: The University of Chicago Press.

Campbell, Lyle (1991): "Some Grammaticalization Changes in Estonian and their Implications". - In: E. C. Traugott, B. Heine (eds.): Approaches to Grammaticalization. Vol. I (Amsterdam, Philadelphia: John Benjamins) (= Typological Studies in Language 19) 285-299.

Cartagena, Nelson (1981): "Sistema, norma y habla del futuro de probabilidad español". - In: H. Geckeler, B. Schlieben-Lange, J. Trabant, H. Weydt (Hgg.): Logos semantikos. Studia linguistica in honorem Eugenio Coseriu. Vol. IV: Grammatik (Berlin, New York, Madrid: De Gruyter) 383-394.

Chafe, Wallace / Nichols, Johanna (eds.) (1986): The Linguistic Coding of Epistemology. - Norwood: Ablex Publishing Corporation (= Advances in Discourse Processes 20).

Comrie, Bernard (1976): Aspect. An Introduction to the Study of Verbal Aspect and Related Problems. - Cambridge, London, New York, Melbourne: Cambridge University Press (= Cambridge Textbooks in Linguistics).

- (1981): "On Reichenbach's Approach to Tense". - In: R. A. Hendrick, C. S. Masek, M. F. Miller (eds.): Papers from the VIIth Regional Meeting. Chicago Linguistic Society 1981 (Chicago: Chicago Linguistic Society) 24-30.

- (1985): Tense - Cambridge, London, New York: Cambridge University Press (= Cambridge Textbooks in Linguistics).

- (1989): "On Identifying Future Tenses". - In: W. Abraham, T. Janssen (Hgg.): Tempus - Aspekt - Modus. Die lexikalischen und grammatischen Formen in den germanischen Sprachen (Tübingen: Niemeyer) (= Linguistische Arbeiten 237) 51-63

Coseriu Eugenio (1976): Das romanische Verbalsystem. Herausgegeben und bearbeitet von Hansbert Bertsch. - Tubingen: Narr (= Tübinger Beiträge zur Linguistik 66).

- (1980): "Aspect verbal ou aspects verbaux? Quelques questions de théorie et de méthode". - In: J. David, R. Martin (éds.): La notion d'aspect. Colloque organisé par le Centre d'Analyse syntaxique de l'Université de Metz 1978 (Metz, Paris: Klincksieck) (= Recherches linguistiques 5) 13-26.

Dahl, Osten (1985): Tense and Aspect Systems. - Oxford: Blackwell.

Damourette, Jacques / Pichon, Edouard (1936): Des mots à la pensée. Essai de Grammaire de la Langue Française (1911-1936). Tome cinquième, verbe (fin): Auxiliaires - Temps - Modes - Voix. - Paris (= Bibliothèque du français moderne, Collection des linguistes contemporains).

Dendale, Patrick (1994): "Devoir épistémique, marqueur modal ou évidentiel?" - In: Langue française 102, 24-40.

- / Tasmowski, Liliane (1994): "Présentation. L'évidentialité ou le marquage des sources du savoir". - In: Langue française 102, 3-7.

Diez, Friedrich (1882): Grammatik der romanischen Sprachen. III, 5. Auflage. - Bonn: Eduard Weber

Fleischman, Suzanne (1982): The Future in Thought and Language. Diachronic Evidence from Romance. - Cambridge, London, New York: Cambridge University Press (= Cambridge Studies in Linguistics).

- (1983): "From Pragmatics to Grammar. Diachronic Reflections on Complex Pasts and Futures in Romance". In: Lingua 60, 183-214

- (1989): "Temporal Distance: A Basic Linguistic Metaphor". - In: Studies in Language 13, 1-50.

Gamillscheg, Emst (1957): Historische franzosische Syntax. - Tübingen: Niemeyer. 
Gobert, David L. / Maisier, Véronique (1995): "Valeurs modales du futur et du conditionnel et leurs emplois en français contemporain". - In: The French Review 68, 1003-1014.

Greenberg, Joseph H. (ed.) (1978): Universals of Human Language. Vol. III: Word Structure. - Stanford: Stanford University Press.

Hablutzel, Ernst (1965): Der Ausdruck des Zukunftigen im Franzosischen. - Winterthur: Keller.

Heger, Klaus (1963): Die Bezeichnung temporal-deiktischer Begriffskategorien im französischen und spanischen Konjugationssystem. - Tübingen: Niemeyer (= Beihefle zur Zeitschrift für romanische Philologie 104).

- (1976): Monem, Wort, Satz und Text. 2., erweiterte Auflage. - Tübingen: Niemeyer (= Konzepte der Sprachund Literaturwissenschaft).

Heine, Bernd / Reh, Mechthild (1984): Grammaticalization and Reanalysis in African Languages. - Hamburg: Buske. - / Claudi, Ulrike / Hunnemeyer, Friederike (1991a): Grammaticalization. A Conceptual Framework. - Chicago, London: The University of Chicago Press.

- / Claudi, Ulrike / Hunnemeyer, Friederike (199Ib): "From Cognition to Grammar: Evidence from African Languages". - In: E. C. Traugott, B. Heine (eds.): Approaches to Grammaticalization. Vol. I (Amsterdam, Philadelphia: John Benjamins) (= Typological Studies in Language 19) 149-187.

Helland, Hans Petter (1994): Sémantique et pragmatique temporelle. Futur simple et futur périphrastique. - Oslo: Universitetet i Oslo (= Historisk-filosofiske fakultet Acta Humaniora).

Herring, Susan C. (1991): "The Grammaticalization of Rhetorical Questions in Tamil". - In: E. C. Traugott, B. Heine (eds.): Approaches to Grammaticalization. Vol. I (Amsterdam, Philadelphia: John Benjamins) (= Typological Studies in Language 19) 253-284.

Herslund, Michael (1989a): "Modality. A Presentation". - In: M. Herslund (ed.): On Modality. Papers from Meetings and Discussions in the Linguistic Circle of Kopenhagen (Kopenhagen: The Circle, Reitzels Boghandel) 7-16.

- (ed.) (1989b): On Modality. Papers from Meetings and Discussions in the Linguistic Circle of Kopenhagen. Kopenhagen: The Circle, Reitzels Boghandel.

Hopper, Paul J. (1979a): "Some Observations on the Typology of Focus and Aspect in Narrative Language". - In: Studies in language 3, 37-64.

- (1979b): "Aspect and Foregrounding in Discourse". - In: T. Givón (ed.): Discourse and Syntax (New York: Academic Press) 213-241.

- (1982a): "Aspect between Discourse and Grammar: An Introductory Essay for the Volume". - In: P. J. Hopper (ed.): Tense - Aspect: Between Semantics and Pragmatics (Amsterdam, Philadelphia: John Benjamins) 3-18.

- (ed.) (1982b): Tense - Aspect: Between Semantics and Pragmatics. - Amsterdam, Philadelphia: John Benjamins.

- (1991): "On Some Principles of Grammaticization". - In: E. C. Traugott, B. Heine (eds.): Approaches to Grammaticalization. Vol. I (Amsterdam, Philadelphia: John Benjamins) (= Typological Studies in Language 19) 15-35.

Imbs, Paul (1960): L'emploi des temps verbaux en français moderne. Essai de grammaire descriptive. - Paris: Klincksieck (= Bibliothèque française et romane. Série A: manuels et études linguistiques 1).

Jacques, Francis (1983): "La mise en communauté de l'énonciation". - In: Langages 70, 47-72.

Kahn, Félix (1954): Le système des temps de l'indicatif chez un Parisien et chez une Bâloise. - Genf: Droz (= Publications romanes et françaises).

Kleiber, Georges / Berthonneau, Anne-Marie (1993): "Pour une nouvelle approche de l'imparfait: l'imparfait, un temps anaphorique méronomique". - In: Langages 112, 55-73.

Klein, Hans-Wilhelm (1980): "Es hat geklingelt; das wird der Brieftrăger sein". - In: Der fremdsprachliche Unterricht $14,140-143$

Koch, Peter (1994): "Gedanken zur Metapher - und zu ihrer Alltäglichkeit". - In: A. Sabban, Ch. Schmitt (Hgg.): Sprachlicher Alltag. Linguistik - Rhetorik - Literaturwissenschaft. Festschrift fur Wolf-Dieter Stempel (Tubingen: Niemeyer) 201-225.

- (1995): "Der Beitrag der Prototypentheorie zur Historischen Semantik: Eine kritische Bestandsaufnahme". - In: Romanistisches Jahrbuch 46, 27-46.

- (1996): "La sémantique du prototype: sémasiologie ou onomasiologie?" - In: Zeitschrift fur franzósische Sprache und Literatur 106, 223-240.

- / Oesterreicher, Wulf (1985): "Sprache der Năhe - Sprache der Distanz. Mündlichkeit und Schriftlichkeit im Spannungsfeld von Sprachtheorie und Sprachgeschichte". - In: Romanistisches Jahrbuch 36, 15-43.

- /Oesterreicher, Wulf (1990): Gesprochene Sprache in der Romania: Franzosisch, Italienisch, Spanisch. - Tubingen: Niemeyer (= Romanistische Arbeitshefte 31). 
- / Oesterreicher, Wulf (1996): "Sprachwandel und expressive Mündlichkeit". - In: Zeitschrift furr Literaturwissenschaft und Linguistik 102, 64-96.

Kortmann, Bernd (1991): "The Triad 'Tense - Aspect - Aktionsart"'. - In: C. Vetters, W. Vandeweghe (eds.): Perspectives on Aspect and Aktionsart. Belgian Journal of Linguistics 6, 9-30.

Laca, Brenda (1996): "Methodische Probleme bei der Erforschung von Grammatikalisierungsphănomenen". - In: S. Michaelis, P. Thiele (Hgg.): Grammatikalisierung in der Romania (Bochum: Universitătsverlag Dr. N. Brockmeyer) (= Bochum-Essener Beiträge zur Sprachwandelforschung 28) 17-48.

Lakoff, Robin (1970): "Tense and its Relation to Participants". - In: Language 46, 838-849.

Langacker, Ronald W. (1977): "Syntactic Reanalysis". - In: C. Li (ed.): Mechanisms of Syntactic Change (Austin, London: University of Texas Press) 57-139.

Lehmann, Christian (1985): "Grammaticalization: Synchronic Variation and Diachronic Change". - In: Lingua e stile 20, 303-318.

- (1995): Thoughts on Grammaticalization. Revised and expanded version. - München, Newcastle: Lincom Europa (= Lincom Studies in Theoretical Linguistics 1).

Lerch, Eugen (1928): "Französische Sprache und französische Wesensart". - In: P. Hartig, W. Schellberg (Hgg.): Handbuch der Frankreichkunde. Erster. Teil (Frankfurt/M.: Diesterweg) 78-146.

Lichtenberk, Frantisek (1991): "On the Gradualness of Grammaticalization". - In: E. C. Traugott, B. Heine (eds.): Approaches to Grammaticalization. Vol. I (Amsterdam, Philadelphia: John Benjamins) (= Typological Studies in Language 19) 37-80.

Ludwig, Ralph (1988): Modalität und Modus im gesprochenen Französisch. - Tubingen: Narr (= ScriptOralia 7).

Mair, Walter N. (1992): Expressivität und Sprachwandel. Studien zur Rolle der Subjektivităt in der Entwicklung der romanischen Sprachen. - Frankfurt/M., Berlin, Bern: Lang (= Europäische Hochschulschriften Reihe 13: Franzosische Sprache und Literatur 178).

Martin, Robert (1981): "Le futur linguistique: temps linéaire ou temps ramifié?" - In: Langages 64, 81-92.

- (1983): Pour une logique du sens. - Paris: Presses universitaires de France.

- (1987): Langage et croyance. Les "univers de croyance" dans la théorie sémantique. - Brüssel: Mardaga (= Philosophie et langage).

Maslov, Yurij S. (1985): "An Outline of Contrastive Aspectology". - In: Y. S. Maslov (ed.): Contrastive Studies in Verbal Aspect (Heidelberg: Groos) (= Studies in Descriptive Linguistics 14) 1-44.

Moens, Marc/ Steedman, Mark (1988): "Temporal Ontology and Temporal Reference". - In: Computational Linguistics 14, 15-28.

Nølke, Henning (1985): "L'illocutoire et sa modalisation". - In: Copenhagen Studies in Language Cebal Series 8, 102129.

- (1989): "Modality and Polyphony - a Study of Some French Adverbials". - In: M. Herslund (ed.): On Modality. Papers from Meetings and Discussions in the Linguistic Circle of Kopenhagen (Kopenhagen: The Circle, Reuthels Boghandel) 45-64.

Osipov, Vladimir (1974): "Grammaticalité au futur antérieur". - In: Le français moderne 42, 20-33.

Palmer, Frank Robert (1986): Mood and Modality. - Cambridge, London, New York: Cambridge University Press (= Cambridge Textbooks in Linguistics).

Parret, Herman (1983): "L'énonciation en tant que déictisation et modalisation". - In: Langages 70, 82-97.

Rauhut, Franz (1957): "Das Futurum exactum als Ausdruck der Vorwegnahme eines spatteren Urteils". - In: G. Reichenkron, M. Wandruszka (Hgg.): Syntactica und Stilistica. Festschrift fur Ernst Gamillscheg (Tubingen: Niemeyer) 421-428.

Reichenbach, Hans (1947): Elements of Symbolic Logic. (Fourth Printing 1952). - New York, London: Free Press.

Roberts, Ian (1993): "A Formal Account of Grammaticalization in the History of Romance Futures". - In: Folia Linguistica Historica 13, 219-258.

Schrodt, Richard (1993): "Von der Diskurssyntax zur Satzsyntax: Reanalyse und/oder Grammatikalisierung in der Geschichte der deutschen Nebensätze". - In: Folia Linguistica Historica 13, 259-278.

Schrott, Angela (1997): Futurităt im Franzősischen der Gegenwart. Semantik und Pragmatik der Tempora der Zukunft. - Tubingen: Narr (= Romanica Monacensia 50).

Schwegler, Armin (1990): Analyticity and Syntheticity. A Diachronic Perspective with Special Reference to Romance Languages. - Berlin, New York: Mouton de Gruyter (= Empirical Approaches to Language Typology 6).

Siewierska, Anna (1991): Functional Grammar. - New York, London: Routledge. 
Smith, Carlota S. (1991): The Parameter of Aspect. - Dordrecht, Boston, London: Kluwer (= Studies in Linguistics and Philosophy 43).

Sperber, Dan / Wilson, Deirdre (1986): Relevance. Communication and Cognition. - Oxford: Blackwell.

Steinmeyer, Georg (1987): "Le futur antérieur comme temps du passé: remarques sur un emploi particulier fréquent du futur antérieur en français". - In: International Review of Applied Linguistics in Language Teaching 25, 119 129.

Sundell, Lars-Göran (1991): Le temps futur en français moderne. - Uppsala: Acta Universitatis Upsaliensis (= Studia Romanica Upsaliensia 49).

Taylor, John R. (1989): Linguistic Categorization. Prototypes in Linguistic Theory. - Oxford: Clarendon Press

Tobler, Adolf (1886): Vermischte Beiträge zur franzősischen Grammatik. 1. Reihe, 37. Futurum exactum an Stelle des Perfectum praesens. - Leipzig: Hirzel.

Togeby, Knud (1982): Grammaire française. Vol. II. Les Formes Personnelles du Verbe. Publié par Magnus Berg, Ghani Merad, Ebbe Spang-Hansen. - Kopenhagen: Akademisk Forlag.

Traugott, Elizabeth Closs (1982): "From Propositional to Textual and Expressive Meanings: Some Semantic-Pragmatic Aspects of Grammaticalization", - In: W. P. Lehmann, Y. Malkiel (eds.): Perspectives on Historical Linguistics (Amsterdam, Philadelphia: John Benjamins) (= Amsterdam Studies in the Theory and History of Linguistic Science Series 4: Current Issues in Linguistic Theory 24) 245-271.

- (1989): "On the Rise of Epistemic Meanings in English: An Example of Subjectification in Semantic Change". - In: Language 65, 31-55.

- (1990): "From Less to More Situated Language: the Unidirectionality of Semantic Change". - In: S. Adamson, V. Law, N. Vincent, S. Wright (eds.): Papers from the 5th International Conference on English Historical Linguistics .Cambridge 6-9 April 1987 (Amsterdam, Philadelphia: John Benjamins) (= Amsterdam Studies in the Theory and History of Linguistic Science Series 4: Current Issues in Linguistic Theory 65) 497-517.

- /Konnig, Ekkehard (1991): "The Semantics-Pragmatics of Grammaticalization revisited". - In: E. C. Traugott, B. Heine (eds.): Approaches to Grammaticalization. Vol I (Amsterdam, Philadelphia: John Benjamins) (= Typological Studies in Language 19) 189-218.

- / Heine, Bernd (1991 a): "Introduction". - In: E. C. Traugott, B. Heine (eds.): Approaches to Grammaticalization. Vol. I (Amsterdam, Philadelphia: John Benjamins) (= Typological Studies in Language) 1-14.

- / Heine, Bernd (eds.) (1991b): Approaches to Grammaticalization. Vol. I, II. - Amsterdam, Philadelphia: John Benjamins (= Typological Studies in Language 19).

- /Hopper, Paul J.(1993): Grammaticalization. - Cambridge: University Press $(\approx$ Cambridge Textbooks in Linguistics)

Ultan, Russell (1978): "The Nature of Future Tenses". - In: J. Greenberg (ed.): Universals of Human Language. Vol. III: Word Structure (Standford: Stanford University Press) 83-123.

Vet, Co (1980): Temps, aspects et adverbes de temps en français contemporain. Essai de sémantique formelle. - Genf: Droz (= Publications romanes et françaises 154)

- (1981a): "Some Arguments against the Division of Time into Past, Present and Future". - In: L. Goossens (Hg.): Bijdragen over semantiek van het 33ste Vlaams Filologencongres. Antwerp Papers in Linguistics 23 (Wilrijk), 153-165.

- (1981b): "La notion de 'monde possible' et le système temporel et aspectuel du français". - In: Langages 64, 109124.

- (1991): "The Temporal Structure of Discourse: Setting, Change and Perspective". - In: S. Fleischman, L. R. Waugh (eds.): Discourse-Pragmatics and the Verb. The Evidence from Romance (London: Routledge) (= Romance Linguistics Series), 7-25.

Wartburg, Walther von / Zumthor, Paul (1947): Précis de syntaxe du français contemporain. - Bern: Francke.

Weinrich, Harald (1982): Textgrammatik der französischen Sprache. - Stuttgart: Klett.

Wilmet, Marc (1976): Etudes de morpho-syntaxe verbale. - Paris: Klincksieck (= Bibliothèque française et romane Série A: Manuels et études linguistiques 34).

Wunderli, Peter (1989): "Le statut précaire de l'aspect verbal". - In: Travaux de Linguistique 18, 73-94. 